\title{
Automated and Standardized Tools for Realistic, Generic Musculoskeletal Model Development
}

Trevor Rees Moon

trmoon@mix.wvu.edu

Follow this and additional works at: https://researchrepository.wvu.edu/etd

Part of the Acoustics, Dynamics, and Controls Commons, Biomechanical Engineering Commons, Computational Neuroscience Commons, Robotics Commons, and the Software Engineering Commons

\section{Recommended Citation}

Moon, Trevor Rees, "Automated and Standardized Tools for Realistic, Generic Musculoskeletal Model Development" (2020). Graduate Theses, Dissertations, and Problem Reports. 7673.

https://researchrepository.wvu.edu/etd/7673

This Thesis is protected by copyright and/or related rights. It has been brought to you by the The Research Repository @ WVU with permission from the rights-holder(s). You are free to use this Thesis in any way that is permitted by the copyright and related rights legislation that applies to your use. For other uses you must obtain permission from the rights-holder(s) directly, unless additional rights are indicated by a Creative Commons license in the record and/ or on the work itself. This Thesis has been accepted for inclusion in WVU Graduate Theses, Dissertations, and Problem Reports collection by an authorized administrator of The Research Repository @ WVU. For more information, please contact researchrepository@mail.wvu.edu. 
Graduate Theses, Dissertations, and Problem Reports

2020

Automated and Standardized Tools for Realistic, Generic

Musculoskeletal Model Development

Trevor Rees Moon

Follow this and additional works at: https://researchrepository.wvu.edu/etd

Part of the Acoustics, Dynamics, and Controls Commons, Biomechanical Engineering Commons, Computational Neuroscience Commons, Robotics Commons, and the Software Engineering Commons 


\title{
Automated and Standardized Tools for Realistic, Generic Musculoskeletal Model Development
}

\author{
Trevor Rees Moon
}

Thesis submitted to the Benjamin M. Statler College of Engineering and Mineral Resources at West Virginia University In partial fulfillment of the requirements for the degree of Master of Science in Mechanical Engineering

\author{
Sergiy Yakovenko, Ph.D., Chair \\ Victor Mucino, Ph.D. \\ Sam Mukdadi, Ph.D. \\ Department of Mechanical and Aerospace Engineering
}

Morgantown, WV

2020

Keywords: motor control, computational neuroscience, musculoskeletal modeling, software, relational database, biomechanics, human leg

Copyright 2020 Trevor Rees Moon 


\begin{abstract}
Automated and Standardized Tools for Realistic, Generic Musculoskeletal Model

Development
\end{abstract}

Trevor Moon

Human movement is an instinctive yet challenging task that involves complex interactions between the neuromusculoskeletal system and its interaction with the surrounding environment. One key obstacle in the understanding of human locomotion is the availability and validity of experimental data or computational models. Corresponding measurements describing the relationships of the nervous and musculoskeletal systems and their dynamics are highly variable. Likewise, computational models and musculoskeletal models in particular are vitally dependent on these measurements to define model behavior and mechanics. These measurements are often sparse and disparate due to unsystematic data collection containing variable methodologies and reporting conventions. To date, there is not a framework to concatenate and manage musculoskeletal data (muscle moment arms and lengths). These morphological measurements need to be assembled to manage, compare, and analyze these data to develop comprehensive musculoskeletal models. Such a framework would enable researchers to select and update the posture-dependent relationships necessary to describe musculoskeletal dynamics, which are essential for simulation of muscle and joint torques in movement. Analogous to all simulations, these models require rigorous validation to ensure their accuracy. This is particularly important for musculoskeletal models that represent high-dimensional, posture-dependent relationships developed from limited and variable datasets. Here, I developed a computational workflow to collect and manage moment arm datasets from available published literature for the development of a human lower-limb musculoskeletal model. The moment arm relationships from multiple datasets were then used to create complete moment arm descriptions for all major leg muscles and were validated within a generic musculoskeletal model. These developments are crucial in advancing musculoskeletal modeling by providing standardized software and workflows for managing high-dimensional and posture-dependent morphological data to creating realistic and robust musculoskeletal models. 


\section{Acknowledgments}

I would first like to thank several people that have guided and supported me throughout graduate school.

First, I would like to thank my advisor, Dr. Sergiy Yakovenko, for his assistance and guidance. I have learned countless skills working with him over the last few years. Some of these include the necessary critical-thinking and management skills to be successful in analyzing and conducting scientific research. I also would like to thank him for his interest and support in pursuing personal ideas and interests as a researcher and in my endeavors. I have learned and grown substantially as a researcher over the years under his supervision.

I would like to thank all my committee members, Dr. Victor Mucino and Dr. Osama Mukdadi, for their help and input on my thesis work.

I would also like to thank all my former and current lab colleagues for all their assistance and support during my time in the Neural Engineering Laboratory. With you, my time in the lab has been a treasured experience. From our random banter around the workspace, lunchtime discussions, and professional criticism and guidance, it has been a pleasure. Specifically, I would like to thank Dr. Matthew Boots, Russell Hardesty, Dr. Anton Sobinov, Ariel Thomas, Olivia Santee, Serhii Bahdasariants, Emily Herrick, and Kacie O'Neill.

Of course, I would like to thank my friends and family for their incredible and ever-lasting support. Without them, this would have not been possible! 


\section{Table of Contents}

Abstract

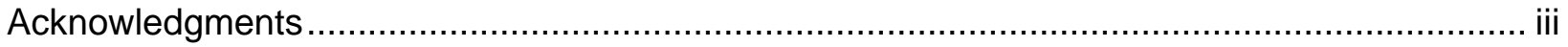

Table of Contents

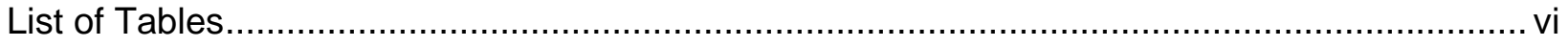

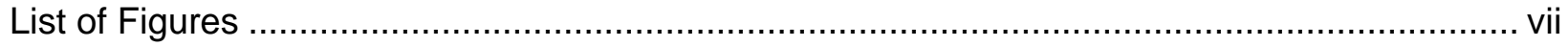

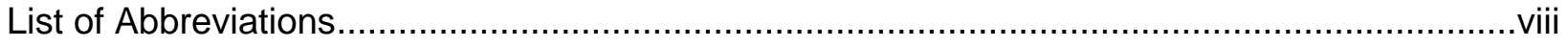

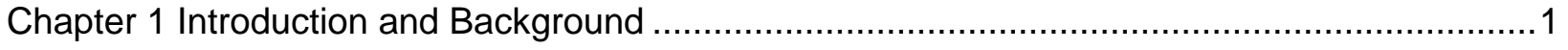

Muscle Recruitment

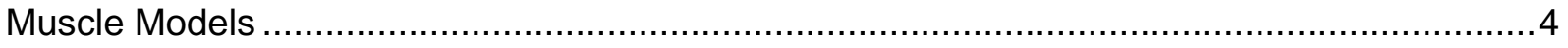

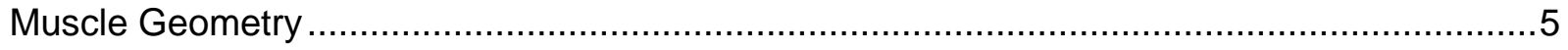

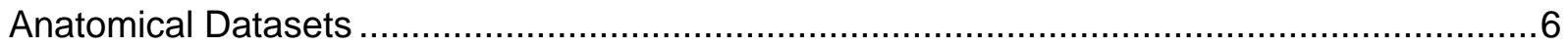

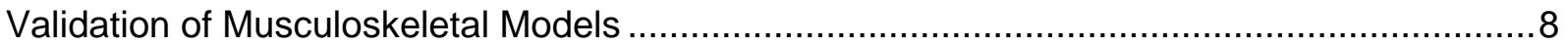

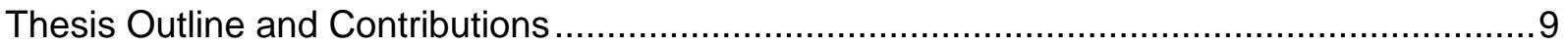

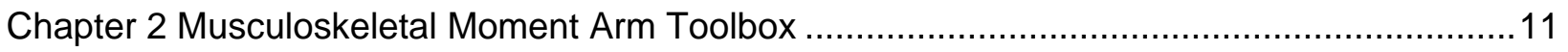

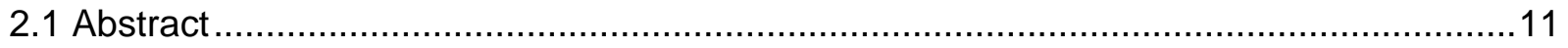

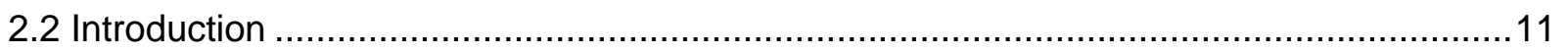

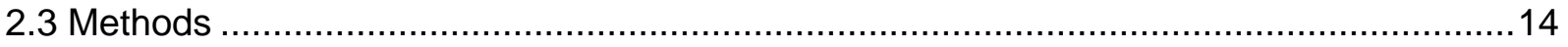

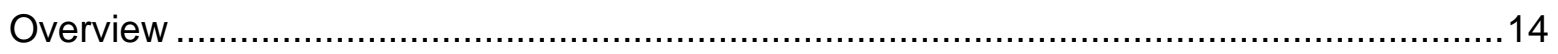

Step 1. Data acquisition and Storage. Database Building Blocks.................................... 15

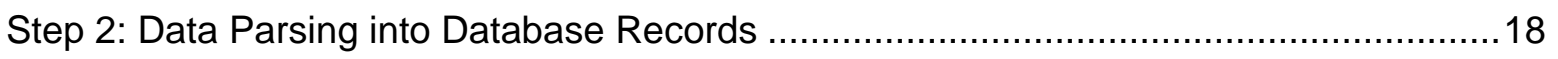

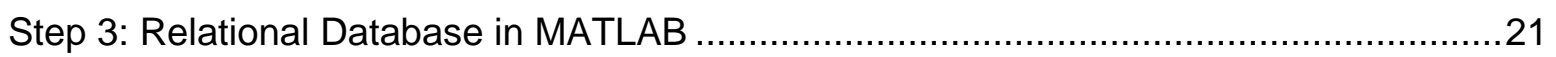

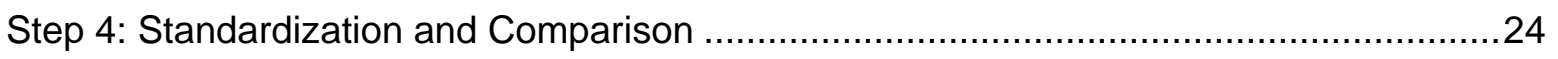

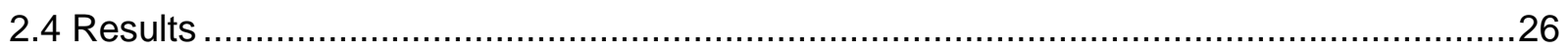

Case Study: Experimental Data for Moment Arm Validation .........................................26

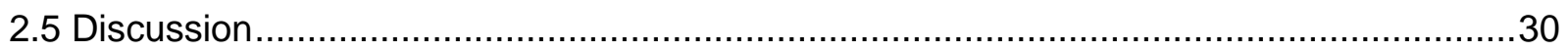

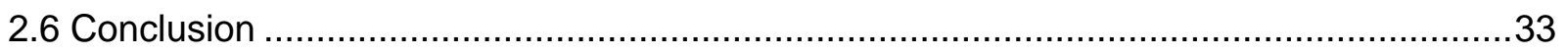

Chapter 3 Validating a Lower-Limb Musculoskeletal Model ................................................ 35

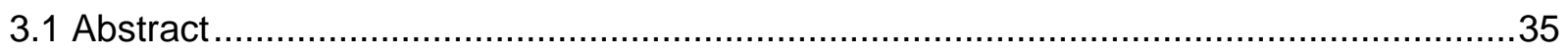

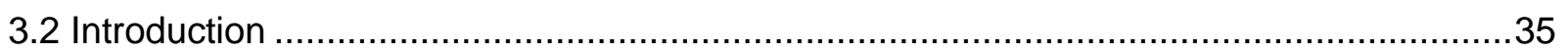

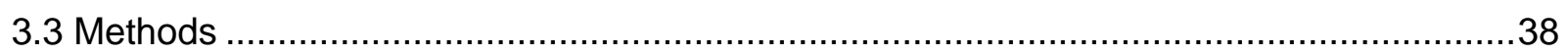

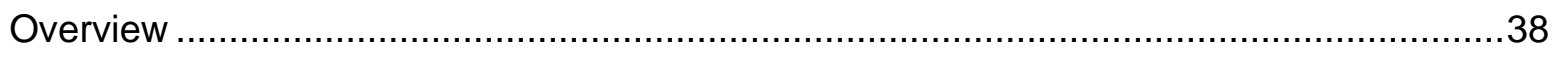

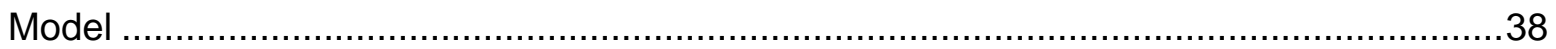




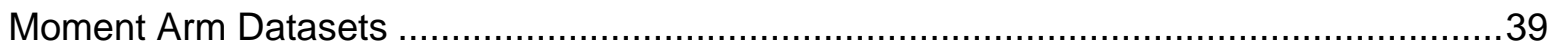

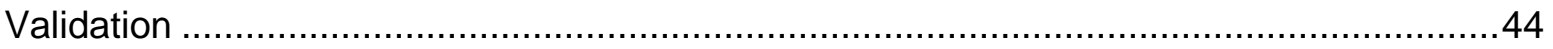

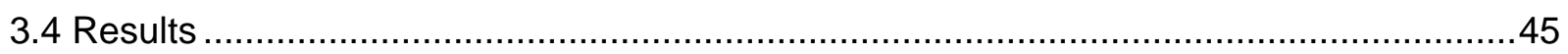

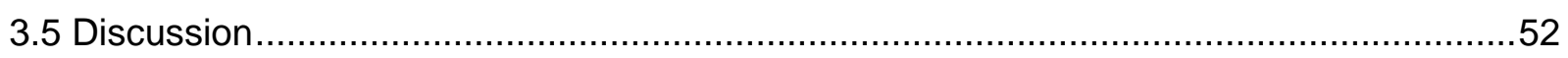

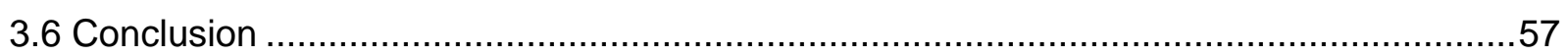

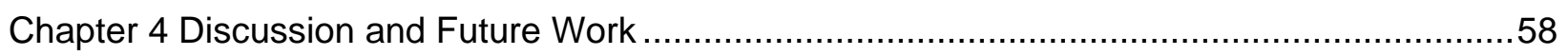

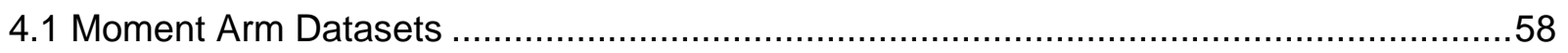

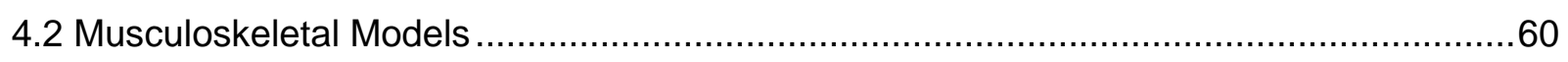

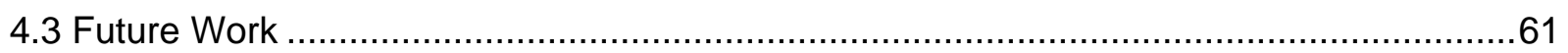

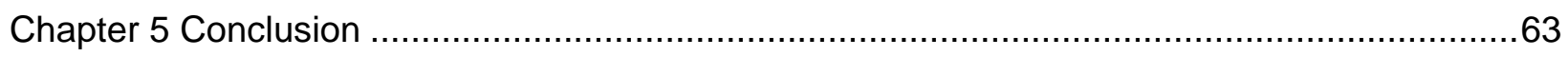

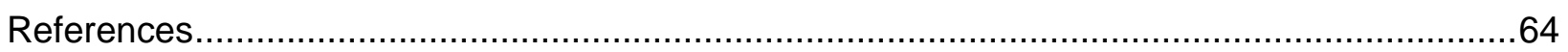




\section{List of Tables}

Table 2.1. Description of dataset parsing group labels and meta labels. The label groups are used to parse various label types and generate Database Fields. Each label group contains a customized parsing action shown in Label Parsing Action.

Table 3.1. Collected published moment arm dataset meta information. 40 


\section{List of Figures}

Figure 2.1. Schematic of the computational workflow. A. General tasks in the processing workflow. B. Specific tasks in the processing pipeline. C. The list of software for each general and specific task(s). 15

Figure 2.2. Schematic of data digitization and file storage description. A. The process of scanning source images and converting them to JSON, TAR, and CSV files. B. The organization of files for a given source. The names in parenthesis indicate the corresponding MATLAB classes created in Step 2. 17

Figure 2.3. Metatables used within the relational database. The metatables shown in green are the required metatables for the database. The moment arm relationships and their meta information are stored in metaDataset and the digitized data are in metaData. The relationship between meta information is shown with a line connecting their common field(s). The fields listed in black are required and those in gray are some commonly added fields. The transparent metatables are some of the optional tables that can be used in the database. .23 Figure 2.4. Standardizing datasets and checking for errors. A. The imported datasets for the moment arms of sartorius for the knee flexion-extension DOF. Each line color corresponds to an individual source and each marker corresponds to an individual dataset within the source. The error in the DOF direction is visible (red lines) when compared to other datasets. $\mathbf{B}$. The same relationships are plotted with the appropriate correction for the imported values.....................26 Figure 2.5 Schematic for combining muscle-DOF relationships for the achilles. A. The workflow used to combine multiple moment arm relationships for the achilles plantarflexiondorsiflexion DOF. B. The extracted relationships from multiple sources. C. The computed standardized profile (in gray) superimposed on the extracted relationships..........................28 Figure 2.6. Computed standardized profiles for muscles at the knee for extension and flexion. A. Semimembranosus knee flexion moment arms. B. Semitendinosus knee flexion moment arms. C. Sartorius knee flexion moment arms. D. Rectus femoris extension moment

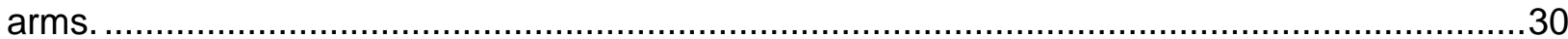
Figure 3.1. Histogram of moment arm zero-crossing locations as a function of ROM. All muscles with moment arm zero-crossings are shown for each muscle-DOF they occur in. Each color corresponds to a DOF (see legend) in the model. .................................................46 Figure 3.2. Categorical comparison of moment arm profiles for all muscles and DOFs. A. The original model (Lai et al., 2017) evaluated for profiles errors. The muscles are labeled vertically and the DOFs are labeled horizontally. The categorical values are shown for each modeled muscle-DOF relationship. B. Examples of the categories used to assess moment arm profile errors.

Figure 3.3. Comparison of measured and simulated moment arms for knee and ankle muscles. A. All available measured moment arm data and created moment arm profile from combining data. B. Comparison of created and simulated moment arms in the validation process. C. Simulated moment arm profiles for multiple OpenSim models. 50 Figure 3.4. Identified muscle path error examples at each joint. The problematic muscle paths are highlighted in red and surrounding muscle paths are opaque. A. The gluteus medius and minimus muscle compartments at 80 degrees hip flexion going through the pelvis. $\mathbf{B}$. The rectus femoris and sartorius muscles at 30 degrees hip extension at the femur. C. The quadriceps (rectus femoris, vastus intermedius, vastus lateralis, and vastus medius) for high knee flexion (90 degrees shown). D. Peroneus (2 left-most) and extensor digitorium/hallicus (2 right-most) muscles in the neutral position at the ankle joint. 52 


\section{List of Abbreviations}

$\begin{array}{ll}\text { CSV } & \text { comma-separated value } \\ \text { DOF } & \begin{array}{l}\text { degree of freedom } \\ \text { electromyography }\end{array} \\ \text { EMG } & \text { JavaScript Object Notation } \\ \text { JSON } & \text { musculotendon unit } \\ \text { MTU } & \text { physiological cross-sectional area } \\ \text { PCSA } & \text { Pearson correlation coefficient } \\ \text { R } & \text { root mean square error } \\ \text { RMSE } & \text { range of motion } \\ \text { ROM } & \text { standard deviation } \\ \text { SD } & \text { tape archive file } \\ \text { TAR } & \end{array}$




\section{Chapter 1 Introduction and Background}

Movement is a vital behavior that allows us to perform actions essential for survival. These movements can vary along a gradient of conscious involvement (e.g., balance and walking) may have a lower cognitive load than reaching or speech. All movements are achieved through the continuous coordination between the nervous and musculoskeletal systems to perform a movement with the desired speed and accuracy. As we age, increases in cognitive demand and muscle weakness (Hunter et al., 2016) decrease our systems operational efficiency, and with increasing life expectancies (Roser et al., 2020), these age-related deficiencies make it more difficult to maintain motor function. Likewise, traumatic loss of motor function from injury or disease can severely decrease a person's quality of life, both physically and psychologically (Graczyk et al., 2018; Laurent et al., 2011). Progressing motor impairments, whether from natural or traumatic causes, can impede the execution of simple tasks and challenge independent living. With the harsh consequences of motor loss, the need to further develop resources to better study, restore, and maintain movement is crucial.

A simple movement can involve one or more joints, each with one or more degrees of freedom (DOFs), and is achieved by selecting appropriate joint kinematics and dynamics to move each segment so that the limb endpoint reaches the desired/final position. This is also referred to as end-effector control. This type of control focuses on the movement goal and the corresponding series of postures (i.e., joint configurations) need to accomplish the task. These series of actions are generated from internal representations of body morphology, including physical limitations due to the range of motion (ROM) and dynamics, also called an internal model. However, limbs (artificial and biological) often have more joints than necessary to achieve desired movements, allowing the same movement to be executed in numerous ways. In motor control, this is often referred to as the degrees of freedom or motor equivalence problem (Bernstein, 1967). This problem encompasses not only kinematic redundancy but also the redundancy due to the larger 
number of muscles than the number of joints they actuate. With the human anatomy containing over 600 muscles and about 250 DOFs, each joint is spanned and actuated by multiple muscles. So how is this task accomplished? One way this question has been studied over the years is through the development of biomechanical models based on experimental motor control studies. These models have been used to gain insight into neural control strategies as tools to help the interpretation of experimental measurements during movement. More notably, these biomechanical models can be used to study how damage to the neuromusculoskeletal system affects control strategies and better facilitate rehabilitation and restoration of motor function.

One type of biomechanical modeling that has become a valuable resource for studying human locomotion is musculoskeletal modeling. These models are instrumental tools for understanding and simulating the transformation from muscle activations to limb dynamics. In other words, musculoskeletal models provide a means for interpreting and transforming our natural command signals to movement. This capability has enhanced our understanding of the underlying characteristics and principles responsible for human locomotion. However, the adoption of these tools outside of the research setting has been hindered by the ability to confidently validate the accuracy and reliability of these models (Boots et al., 2020). Moreover, the musculoskeletal datasets used to describe the underlying properties and mechanics of these models are sparse and disparate, which are vital to achieving accurate and robust simulations (Boots et al., 2020; Cook et al., 2014; Rajagopal et al., 2016). Although verification and validation are a common and necessary practice for computer simulations, there is no standard protocol for developing and validating biomechanical models. Likewise, there are large efforts to create standardized software and resources for expanding and maintaining biological datasets. Thus, developing accurate models must lead to the creation of vital tools for the study and rehabilitation of locomotion. I have developed an intuitive, step-by-step workflow with automated software tools to manage musculoskeletal datasets (see chapter 2) that is used to compare and analyze generic moment 
arm relationships for the human lower-limb in current musculoskeletal models (see chapter 3). The following sections describe the applications, background, and validation of musculoskeletal models.

\section{Muscle Recruitment}

When we decide to move, cortical commands descend the spinal cord to the neural circuitry that generates motor outputs to muscles. The output cells are called motoneurons; they innervate muscle fibers within a muscle. Multiple muscle fibers innervated by the same motoneuron are called a motor unit, and numerous motor units innervate each muscle. These units can be categorized into two different groups based on the number and type of muscle fibers they innervate. The first group is the motor units that innervate Type I (i.e., slow-twitch) muscle fibers. These slow-twitch fibers are slow contracting and produce weak forces; however, they are fatigue resistant due to their aerobic behavior. The second group of motor units innervates Type II (i.e., fast-twitch) fibers. These fast-twitch fibers are often divided into two groups: Type Ila and Type Ilb with a range of contraction speed and force production. These fibers are, however, fast fatigable. It is not surprising then that the system activates different unit types following a recruitment order that balances the trade-off between force production and muscle fatigue. This order is formulated as Henneman's size principle, which describes the gradual recruitment from slow to fast motor units (Henneman, 1957). The recruited motor units also undergo modest changes in the firing rate to increase force generation. This recruitment order allows the central nervous system to efficiently generate force to match task demands. In musculoskeletal modeling, muscle recruitment is often represented by via electromyography (EMG). These signals are usually band-pass filtered to remove nonphysiologically low and high frequencies, rectified, lowpass filtered, and normalized (e.g., EMG linear envelope). This signal is further used to simulate force generation with Hill-type muscle models within feedforward or inverse dynamics simulations (Erdemir et al., 2007; F.E. Zajac, 1989). 


\section{Muscle Models}

Musculoskeletal models represent musculotendon systems within body segmental mechanics. These muscle force generation models are used with the equations of motion to evaluate system state. The description of muscle force generation is founded on years of experimental paradigms and observations, which have led to several iterations or types of muscle models over the years. In biomechanical analyses, Hill-type muscle models are the most common (Romero \& Alonso, 2016). The classical work by A.V. Hill (Hill, 1938) expanded the Kelvin structural model by adding a "contractile" element in series with the viscoelastic element (Romero \& Alonso, 2016; Yamaguchi, 2001). The added contractile element accounted for the force produced by the sliding interactions of myofilament proteins (e.g., actin and myosin) within the sarcomere described by sliding filament theory (Huxley, 1957). Briefly, Huxley's theory describes force-generation at the level of actin-myosin interactions within a muscle fiber unit, called a sarcomere. The binomial distribution of force-generation is relative to the overlap of actin and myosin proteins and the formed actin-myosin complexes (i.e., cross-bridges). The number of cross-bridges formed during contraction is directly related to the force produced and is determined by the number of available actin-myosin binding sites and the speed of cross-bridge attachment and detachment. The time constants of the attachment and detachment process (i.e., cross-bridge cycle) define the muscle force-velocity relationship. The muscle force-length characteristic arises from the overlapping of actin and myosin filaments, which is dependent on sarcomere length. The remaining mechanical elements of the Hill-type muscle model account for the passive forces produced by stretched connective tissue and the tendon connecting muscle to bone. Depending on the simulated task, the tendon could be assumed to be infinitely stiff (i.e., tendon compliance) to simplify computational load without loss of accuracy (F.E. Zajac, 1989). Each musculotendon can then be defined by the selection of appropriate morphological measurements that describe and/or define the force-length and force-velocity relationships. For example, the assumption that all muscle 
fibers are the same length and type simplifies the parameters needed to define the muscle forcegeneration capacity; the maximum isometric force a muscle produces can be estimated as the product of physiological cross-sectional area (PCSA) and specific tension. By normalizing these relationships for the muscle and tendon's operational ranges, the force-length and force-velocity characteristics become dimensionless and be scaled by measurements to represent specific musculotendon dynamics. The selection of these parameters and their assumptions provide the structural and dynamic differences seen across individual muscles and published muscle models (Millard et al., 2013; Thelen, 2003; van Soest \& Bobbert, 1993; F.E. Zajac, 1989).

\section{Muscle Geometry}

In conjunction with muscle recruitment, muscle geometry plays an equally crucial role in muscle dynamics. Regarding Hill-type muscle models, muscle geometries would define musculotendon parameters such as muscle fiber orientation (i.e., pennation angle) and PCSA. These specific values would define the maximum amount of force a given muscle could produce with the given muscle morphology and size. Muscle geometry also refers to the defined muscle paths that describe the length of the musculotendon actuator for any given posture. These paths are represented in three-dimensional space with a set of points placed relative to anatomical bone landmarks that wrap and slide over joint-specific geometries. These points also describe the effective attachment sites (e.g., muscle origin and insertion locations) and geometry around neighboring muscle, tendon, and joints. Moreover, these points inherently determine the length of the musculotendon in each posture and therefore indicate where on the muscle model's forcelength and tension-length curves the muscle is operating.

The known muscle lengths and force-generation capacity of muscle are then scaled by the muscle's moment arm defined by the path geometry around the joint to produce the posturedependent torques during movement. Thus, it is of high importance to carefully consider the effects of selecting these geometric features and parameters. Typically, measurements are 
performed on cadavers (e.g., Haugstvedt et al., 2001; Loren et al., 1996). These efforts aim to benefit musculoskeletal modeling by providing additional information about inter-individual anatomical variability. Recently, these efforts have employed modern imaging and simulation techniques (e.g., Monte Carlo Analysis) to better explore and quantify anatomical variability in several parameters like attachment sites and the number of via (intermediate) points used to define muscle paths (Bujalski et al., 2018; Carbone et al., 2012; Duda et al., 1996; van der Helm \& Veenbaas, 1991). Likewise, the introduction of statistical or probabilistic modeling has expanded this area of musculoskeletal modeling by identifying expected observed variations in anatomical features among individuals (Nolte et al., 2016), which can be used to better generate subject-specific models. Furthermore, one less studied component is the overall effect these changes impose of computed moment arms, despite quantifying the effect on overall model predictions (e.g., joint torques). Due to the direct relationship of moment arms on simulated torques, it is imperative to accurately model underlying moment arm relationships by ensuring the muscle paths defining them are physiologically valid. Yet, there is limited available data describing the necessary moment arm relationships needed to compare and validate simulated values.

\section{Anatomical Datasets}

The outcome of musculoskeletal modeling predictions is crucially dependent on the morphological datasets defining the underlying fundamental model parameters and dynamics. One inherent challenge is composed of the nature of musculoskeletal modeling, which aims to model and simulate a biological system that contains inherent variability amongst individuals (Cook et al., 2014). Therefore, it is expected that these data describing biological parameters and relationships are, in general, highly variable. Furthermore, experimentally determined data are incredibly sparse and disparate, leading researchers to combine select datasets to fully-describe musculoskeletal dynamics that could contain physiological non-linearities (Goislard De Monsabert et al., 2018). Additional variability in these experimental datasets are attributed to the innate 
differences in experimental paradigms and methodologies, which alone can lead to large errors reported within and across datasets. In the context of muscle moment arms, several factors contribute to these differences.

One source of error between moment arm data is the measurement technique. There are several different methods for calculating or measuring moment arms (see reviews in (An et al., 1984; Klein et al., 1996). One method is the center of rotation (COR) or graphical method (Reuleaux, 1876). This approach is based on geometric measurements of $2 \mathrm{D}$ digital scans from computed tomography $(\mathrm{CT})$, ultrasound, or magnetic resonance imaging (MRI). These scans are used to define the muscle's line of action and the joint's COR to reconstruct the 3D moment arms. This method has been used in cadaveric (Dostal et al., 1986; Walter Herzog, 1992; Spoor \& van Leeuwen, 1992) and in vivo specimens (Constantinos N. Maganaris, 2004; Constantinos N. Maganaris et al., 1998; Constantinos N Maganaris et al., 1999; Nemeth \& Ohlsen, 1985, 1989; Rugg et al., 1990; Wretenberg et al., 1996). However, determining the line of action in large tendons and the orientation of the joint's axis of rotation can be difficult (Klein et al., 1996). Furthermore, this method is limited by the resolution and cost of operation of medical imaging devices.

An alternative is direct load measurements, which use the principle of moment equilibrium around a joint. By applying an external force of known magnitude to the muscle of interest (typically done in cadavers) and measuring the resistive force at the distal point on the segment, the moment arm is calculated from fulcrum equations (An et al., 1984). This methodology is suitable for simple joints (e.g., knee) that can be easily isolated and externally loaded (i.e., the quadriceps) (Draganich et al., 1987; Grood et al., 1984; Visser et al., 1990; Yamaguchi \& Zajac, 1989). Like the graphical approach, this method suffers from errors if the axis of rotation is not accurate.

The last, and perhaps most popular, method commonly used to determine moment arms is the tendon excursion (TE) or joint displacement method (An et al., 1983; Landsmeer, 1961). Based 
on the principle of virtual work, this approach defines the moment arm as the slope of tendon excursion (i.e., displacement) and joint displacement. In other words, given some applied external moment to the distal segment, the resulting tendon displacement is assumed to be caused by the rotation of the joint, and the moment arm for the specific posture would become the change in tendon and joint displacement (i.e., slope) (An et al., 1983). The advantage of this method is that it does not require determining the location or orientation of the axis of rotation like alternative methods and can be performed easily for the muscle's entire range of motion; however, the major drawback is that this method is only capable of measuring in-plane moment arms. Nonetheless, the popularity of this method is likely due to its simplicity for not requiring the axis COR and has been used to measure moment arms at every joint in the human lower extremity (A. S. Arnold et al., 2000; A. S. Arnold \& Delp, 2001; W.L. Buford et al., 1997; Scott L. Delp et al., 1994; Scott L Delp et al., 1999; Klein et al., 1996; McCullough et al., 2011; Spoor et al., 1990).

It is becoming increasingly common to compare several of these methods within the same study, which allows more reliable comparisons between selected techniques (Fath et al., 2010; Constantinos N. Maganaris, 2004); moreover, the inclusion of additional measurement conditions (e.g., maximum voluntary contraction trials) assist in determining how moment arms change with different levels of effort (Ito et al., 2000; Lee \& Piazza, 2008; Constantinos N. Maganaris, 2004). Still, these data only account for the necessary moment arm relationships that should be represented by muscle path geometry to compute muscle torques.

\section{Validation of Musculoskeletal Models}

Valid musculoskeletal models that accurately and robustly simulate the human neuromusculoskeletal system has been the focus of recent research. These models provide a non-invasive alternative for simulating varying pathological abnormalities and conditions to design better treatment and rehabilitation techniques. While the volume of computational and treatment studies continues to increase, standardized validation metrics and processes have yet to be 
developed. Other intensive computational and simulation-based disciplines have well-established protocols for developing and verifying model accuracy (e.g., biomechanics of heart muscles); yet, musculoskeletal modeling has to develop the same computational standards (Hicks et al., 2015). The main limitation of progress is the availability of the experimental morphological datasets containing the structural and functional relationships used to develop these models. Currently, there are no rigorous validation benchmarks to ensure that the underlying, posture-dependent relationships are physiologically appropriate when simulating movement.

\section{Thesis Outline and Contributions}

Here, I developed a computational workflow to collect and manage moment arm datasets from available published literature for the development of a human lower-limb musculoskeletal model. The moment arm relationships from multiple datasets were then used to create complete moment arm descriptions for all major leg muscles and were validated within a generic musculoskeletal model. My work aimed to develop automated and standardized tools to acquire and manage the high-dimensional, posture-dependent moment arm datasets used to develop and validate generalizable musculoskeletal models.

In chapter 2, I describe the main limitations of musculoskeletal datasets, specifically moment arms, and the lack of standardized tools and procedures used to collect and manage these datasets for musculoskeletal models. The goal was to create tools that would allow users to easily and quickly generate a database for musculoskeletal datasets. Here, I developed an intuitive stepby-step process for extracting moment arm relationships from scientific literature and automated tools to create custom, relational databases from extracted datasets. The main contribution of this work is a standardized computational workflow (with automated tools) that allows users to extract and manage musculoskeletal datasets for comparison and analysis.

In chapter 3 , the goal was to generate generic moment arm profiles to develop and validate a generalizable musculoskeletal model. I utilized multiple moment arm datasets from a custom 
database to create generic muscle-DOF relationships for the human lower-limb. The created moment arm profiles were used in a validation process to evaluate structural and functional errors in moment arms and muscle paths for a musculoskeletal model (Boots et al., 2020). Also, I compared the simulated moment arm profiles of several other published OpenSim musculoskeletal models. The impact of this work was the use of generic moment arm relationships to assess structural and functional errors in current lower-limb musculoskeletal models, as well as highlight and recommend areas of future work to move towards fully-described and validated lower-limb models.

In chapter 4, I present a general discussion overviewing chapters 2 and 3 and future work.

In chapter 5, I review the overall contributions of the thesis and present concluding remarks. 


\section{Chapter 2 Musculoskeletal Moment Arm Toolbox}

\subsection{Abstract}

Human movement is a challenging task determined by the complexity of the musculoskeletal system and its interactions with the environment. The key obstacle in our theoretical understanding of the body's control mechanism is the lack of valid models describing the realistic mechanical actions of muscles. The corresponding empirical measurements of muscle mechanics are sparse and distributed through a body of scientific literature with varied methodologies, limb morphologies, and reporting conventions. Here, we developed a computational framework to assist in the development and comparison across published datasets for the description of muscle moment arms, which are essential posture-dependent parameters for estimating muscle torques. The solution is a combination of tools that digitize and collate disparate measurements within a relational database. This framework is an essential tool for improving the quality of musculoskeletal modeling.

\subsection{Introduction}

The desire to understand the human anatomy and physiology and its role in movement has spanned dozens of generations in the medical and scientific community. Previous efforts in these fields have gathered invaluable insights and data about the fundamental principles governing locomotion. In particular, the progression of musculoskeletal modeling, the branch of modeling muscular and skeletal dynamics and morphology, has largely benefited as a result. These models have been used to simulate muscle coordination during gait (A. S. Arnold et al., 2007), joint contact forces (DeMers et al., 2014; Modenese et al., 2018; Richards et al., 2018), balance (Chvatal \& Ting, 2013), and propulsion (Hamner et al., 2010; Neptune et al., 2001). These models also provide a non-invasive alternative to better study underlying muscle properties and architecture that are not generally accessible. Expanding on the simulations of normal and healthy 
conditions, these models are easily modifiable to simulate pathological conditions seen in cerebral palsy (A. S. Arnold \& Delp, 2001) or muscle weakness in elderly adults (van der Krogt et al., 2012). Likewise, they can serve as an invaluable tool for clinicians and surgeons to help diagnosis and develop treatment plans (Scott L. Delp et al., 1994; Magermans et al., 2004; Piazza et al., 2001), restoration of lost limb function through muscle reinnervation (He Huang et al., 2008; Kuiken, 2009), and real-time human-machine interfaces (Boots et al., 2020; Crouch \& Huang, 2016; Durandau et al., 2018; Sartori et al., 2018). The rapid development of musculoskeletal models has been attributed to their nature to model the relationships necessary to describe movement and the further development of standardized software tools.

The increase of musculoskeletal modeling is in large part to the contribution of open-source modeling and simulation software such as OpenSim (Scott L. Delp et al., 2007; Seth et al., 2018), SIMM (Scott L. Delp \& Loan, 1995), and AnyBody (Damsgaard et al., 2006). These tools provide an instrumental framework for designing custom musculoskeletal models and simulations that promote a shared distribution of tools through plug-ins and extensions. Software for processing motion capture data (Burger \& Toiviainen, 2013; Mantoan et al., 2015), rapid prototyping of subject-specific models (Valente et al., 2017), finite element analysis (Maas et al., 2012), and optimization solvers (Pizzolato et al., 2015) are common additions to existing musculoskeletal tools; however, there are less open-source tools catered to musculoskeletal model development and validation. Such tools include scaling musculoskeletal models to subject-specific parameters (Winby et al., 2008) or using non-invasive imaging techniques (e.g., MRI, ultrasound) to determine these specific parameters (Nolte et al., 2016). Consequently, the accelerated growth of these tools has overshadowed the lack of standardized verification and validation procedures (Henninger et al., 2010; Hicks et al., 2015; Lund et al., 2012).

A substantial consideration when developing musculoskeletal models is their crucial dependency on anatomical and physiological data used to describe their underlying relationships. These data 
are often sparse and variable in and across scientific literature (Goislard De Monsabert et al., 2018), which is commonly observed when modeling biological systems (Cook et al., 2014). However, it has been only recently that some of these datasets have become more comprehensive and suitable for generic musculoskeletal modeling. Moreover, these data are often not readily available and only attainable from the available scientific literature. Acquiring adequate literature sources containing the necessary muscle model (Handsfield et al., 2014; Ward et al., 2009) or muscle geometry (Carbone et al., 2015) data (e.g., muscle moment arms) to describe neuromusculoskeletal dynamics is inefficient and a significant limitation in the time required to develop these models. Additionally, manual digitalization of these data can be tedious and susceptible to errors (i.e., manually extracting data points from a line plot). To overcome these issues, one must use additional digitization software tools (Huwaldt, 2018; jiro, 2020; Rohatgi, 2019) to extract data from scanned images (e.g., literature figures) to store and use the data. Then, it would be recommended to store the digitized data into some structured file hierarchy, flat files (e.g., tables) or relational database for convenient access to the data. Although there are several solutions for each aspect of the described process, we currently have not identified a complete and integrated framework for musculoskeletal datasets.

In our study, we developed a data collection and validation framework streamlined to generate a posture-dependent dataset of musculoskeletal measurements for the development of accurate biomechanical models. We have combined and developed open-source tools for the digitization of published relationships and a custom relational database to accumulate and compare these measurements. Here, we demonstrate the use of this computational pipeline for the generation of human lower-limb models. 


\subsection{Methods}

\section{Overview}

The musculoskeletal datasets for biomechanical models are published in multiple sources with reported measurements collected with variable methods and over limited and varied posture domains. These diverse and sparse measurements are notoriously difficult to work with. For this reason, we have developed the full process of dataset generation outline in four consecutive steps (Figure 2.1): 1) data acquisition and storage, 2) dataset processing, 3) database creation, and 4) standardization (muscle naming and posture conventions), comparison across sources, and analysis. The moment arm database package was developed to provide tools to interact with digitized moment arm data to create a database. The package includes several MATLABimplemented classes to automate the conversion of exported WebPlotDigitizer files and autogenerate metatables containing the moment arm relationships. These data files are stored in a series of parent-child directories containing individual moment arm "datasets" describing various

muscle-DOF relationships (see Step 1 and Step 2). Extracting all these data into structured datasets is achieved by iterating all the file directories and parsing the data into unique muscleDOF relationships based on the moment arm data and associated metadata (Step 3). The resulting unique moment arm datasets may then be exported as metatables to a custom relational database (see Step 4). 
A. General Process

B. Processing Steps C. Software

\begin{tabular}{|c|c|c|}
\hline $\begin{array}{l}\text { Data Acquisition } \\
\text { and File Storage }\end{array}$ & $\begin{array}{l}\text { Scan figures and tables } \\
\downarrow \\
\text { Figure } \\
\downarrow \\
\text { WebPlotDigitizer } \\
\downarrow \\
\text {.json / .tar files } \\
\text { Store file data }\end{array}$ & \\
\hline Dataset Processing & $\begin{array}{c}\text { Pull data from files } \\
\text { Concatenate all data } \\
\text { Parse full dataset } \\
\text { Create unique datasets }\end{array}$ & $\begin{array}{l}\text { webplotdigitizer } \\
\text { metatable, } \\
\text { dataset, dbdir, } \\
\text { refdir, dofdir, } \\
\text { labelparser }\end{array}$ \\
\hline Database Creation & $\begin{array}{c}\text { Create database } \\
\text { Create metatables } \\
\downarrow \\
\text { Import metatables }\end{array}$ & $\begin{array}{l}\text { metatable, } \\
\text { database, } \\
\text { dataset }\end{array}$ \\
\hline $\begin{array}{l}\text { Standardization and } \\
\text { Analysis }\end{array}$ & $\begin{array}{c}\text { Check for errors } \\
\downarrow \\
\text { Standardize datasets } \\
\downarrow \\
\text { Compare data }\end{array}$ & \\
\hline
\end{tabular}

Figure 2.1. Schematic of the computational workflow. A. General tasks in the processing workflow. B. Specific tasks in the processing pipeline. C. The list of software for each general and specific task(s).

\section{Step 1. Data acquisition and Storage. Database Building Blocks}

\section{Importing}

Moment arm data is often presented in literature figures which are not readily analyzable and must be digitized to a usable format before any analyses can be conducted. We used WebPlotDigitizer, an available open-source tool (Rohatgi, 2019), to accurately digitize the relationships between moment arms and limb posture. This software supports automatic scaling for various axes types used for storing moment arm data (e.g., 2D line and bar plots). With automatic and manual detection tools, extracted data points are exported as common data files (e.g., JavaScript Object Notation (JSON) or Tape Archive (TAR)). The developed package includes standalone functions and classes to import and edit these data relationships. Importing the data into the workspace can be done with functions readjson and readtar for each data file 
type. These functions create a custom dataset struct variable in the MATLAB workspace with fields corresponding to the organization in the exported JSON and TAR files. The dataset structure has four fields containing the following details: 1) axes scaling and type (axescoll), 2) empirical data (datasetColl), 3) additional measurements (measurementColl), and 4) software version (version). These fields are classes containing the data and meta information for the scanned image's axes, extracted pairs of moment arm and posture values, additional measurements (e.g., the distance between points), and version, respectively. An additional option is to load the data file as a wpd object, which is a composition class comprised of axescoll, datasetColl, measurementColl, and version objects. The collection objects are instances (i.e., specific implementations) of the abstract collection class that accesses individual axes, dataset, and measurement objects extracted from the scanned image. Each collection class also includes appropriate methods for accessing and computing extracted data and metadata. For moment arms, this enabled us to automatically extract posture-dependent values from dataset objects and match these data to their corresponding DOF joint angles and sign conventions (e.g., hip flexion is a positive value) stored in axes objects. 

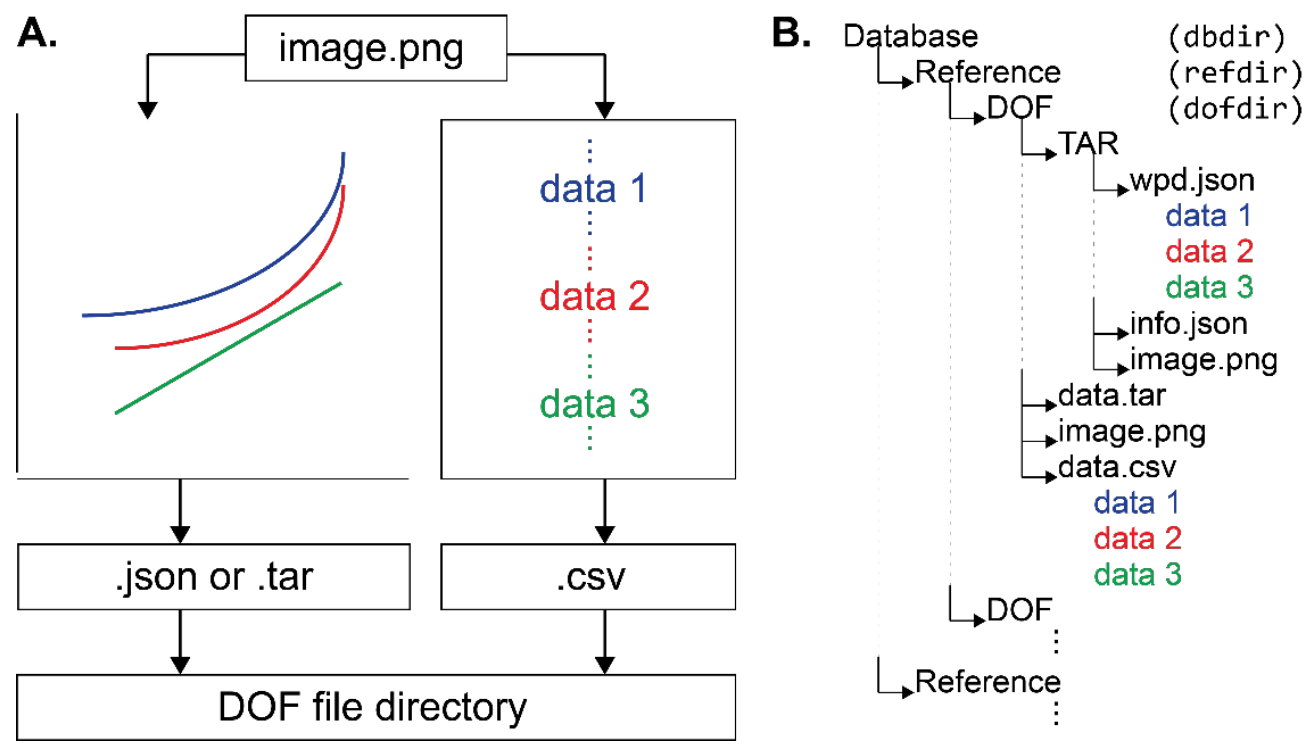

Figure 2.2. Schematic of data digitization and file storage description. A. The process of scanning source images and converting them to JSON, TAR, and CSV files. B. The organization of files for a given source. The names in parenthesis indicate the corresponding MATLAB classes created in Step 2.

\section{Dataset Nomenclature}

Individual muscle-DOF relationships extracted from WebPlotDigitizer were given custom labels that associated the data with corresponding meta information. For example, a scanned image could not only generate the average and/or individual profiles for different postures but also the relevant statistics (i.e., standard deviation). Since multiple profiles could be extracted from a single source image, a unique string label was required to reference individual measurements within a given source and/or muscle. The unique label contained the muscle name followed by additional meta labels separated with an underscore. The additional meta labels can represent individual muscle compartments or trial conditions (e.g., measurements during maximum voluntary contractions). For example, the label GLUTMAX_ANTERIOR_MVC30 corresponds to data for the anterior compartment of gluteus maximus during a 30\% maximal voluntary contraction trial. This identifier associated both measurements and the corresponding meta information (see Step 2). The portion of the label describing the muscle matched the original used in the source. Differences in this part of the label were reconciled after importing the data into the relational database (see Step 4). 


\section{Directory File Organization}

Digitized moment arms were stored in a hierarchy of parent-child file directories with the highest level (Database) containing all the measurements (see Figure 2.2B). The next level (Reference) stored the data for each DOF from scanned source figures and tables. Each DOF was uniquely identified with a custom label for each source study (like the labels used for muscles) and were consistent with those used in Step 4. Each DOF directory contained measurements (TAR files), source images (PNG file in TAR archive file, see Figure 2.2A), or source tables (CSV file). Each source could provide one or multiple DOF directories from scanned figures, with each figure capable of containing multiple moment arm profiles shown in Figure 2.2A. The output from WebPlotDigitizer was a TAR file. Although WebPlotDigitizer can also export CSV or JSON files, we implemented our solution around the TAR file because it contains all the necessary images and data needed to share the extracted contents. Moreover, the exported JSON and TAR files are easily incorporated into automated workflows with standard programming software (e.g., MATLAB and JavaScript). This organization allowed us to effortlessly import extracted datasets from multiple sources into a relational database.

\section{Step 2: Data Parsing into Database Records}

\section{Directories}

The directory file organization was described using four classes. One generic class (database.directory) described all the methods and properties used in the three specific classes (Fig. 2.2B). These subclasses described the following file hierarchical levels: 1) top-level file directory (dbdir), 2) source (refdir), 3) DOF information (dofdir). The abstract superclass was used to define common directory operations like finding child directories and files. In addition to basic directory operations, the class also included methods and properties associated with the dataset class so that the implemented directory objects could modify the field names and properties used in the returned dataset. Each specific database.directory subclass (dbdir, refdir, dofdir) implemented a getData method that was responsible for retrieving the moment 
arm data from its respective level in the file directory hierarchy. The getData method operates on the DOF level (dofdir.getData) to recursively collect all available relationships. For example, refdir.getData would locate the first DOF directory under the "Reference" directory and call dofdir.getData, which would return a dataset object containing all the extracted muscle-DOF relationships. The refdir.getData method repeats this process for every other DOF directory until all directories were collected and concatenated in dataset.

\section{Dataset Class Implementation}

The dataset class was implemented with fields corresponding to six required dataset properties: 1) muscle label (sMuscle), 2) DOF angle value (nDOF), 3) moment arm value (nMA), 4) standard deviation (nSD), 5) unique DOF name (sDOF), and 6) the source reference (sRef). Additional data fields were used for metadata and were often generated when parsing the meta labels. A dataset object is like a metatable that can be manipulated to add, remove, and edit rows and additional meta columns. The implementation provides a standardized data structure for concatenating multiple measurements and preparing them for parsing and database importing procedures.

Table 2.1. Description of dataset parsing group labels and meta labels. The label groups are used to parse various label types and generate Database Fields. Each label group contains a customized parsing action shown in Label Parsing Action.

\begin{tabular}{|c|c|c|c|}
\hline Label Group & Label Description & Label Parsing Action & Database Fields \\
\hline sd & $\begin{array}{l}\text { standard deviation } \\
\text { value }\end{array}$ & $\begin{array}{l}\text { Transfers sd data to the } \\
\text { corresponding records nSD field }\end{array}$ & NA \\
\hline general & non-specific label & $\begin{array}{l}\text { Creates a field with 'b' + the } \\
\text { label name in all uppercase }\end{array}$ & Boolean field \\
\hline compartment & muscle compartment & $\begin{array}{l}\text { Creates fields 'b' and 's' + } \\
\text { 'COMPARTMENT'. The label } \\
\text { name is placed in } \\
\text { 'sCOMPARTMENT' }\end{array}$ & $\begin{array}{l}\text { Boolean and string } \\
\text { fields }\end{array}$ \\
\hline count & $\begin{array}{l}\text { the label contains } \\
\text { numeric data or } \\
\text { specifier (e.g., subject } \\
\text { 1) }\end{array}$ & $\begin{array}{l}\text { Creates fields 'b' and ' } n \text { ' + the } \\
\text { label name in all uppercase }\end{array}$ & $\begin{array}{l}\text { Boolean and numeric } \\
\text { fields }\end{array}$ \\
\hline dof & $\begin{array}{l}\text { the label specifies } \\
\text { moment arm data is }\end{array}$ & $\begin{array}{l}\text { Creates fields 'b', ' } n \text { ', and 's' + } \\
\text { 'OUTDOF'. The label name is }\end{array}$ & $\begin{array}{l}\text { Boolean, numeric, } \\
\text { and string fields }\end{array}$ \\
\hline
\end{tabular}




\begin{tabular}{|c|c|c|c|}
\hline & $\begin{array}{l}\text { not measured DOF } \\
\text { neutral }(0 \text { deg. }) \\
\text { position }\end{array}$ & $\begin{array}{l}\text { placed in 'sOutDOF' and } \\
\text { numeric data (if any) in } \\
\text { 'nOutDOF' }\end{array}$ & \\
\hline scaling & $\begin{array}{l}\text { simulated moment arm } \\
\text { from a scaled model }\end{array}$ & $\begin{array}{l}\text { Creates fields 'b' and 's' + } \\
\text { 'SCALED'. The label name is } \\
\text { placed in 'sSCALED' }\end{array}$ & $\begin{array}{l}\text { Boolean and string } \\
\text { fields }\end{array}$ \\
\hline region & $\begin{array}{l}\text { non-standard deviation } \\
\text { error or bound value }\end{array}$ & $\begin{array}{l}\text { Calculates standard deviation } \\
\text { from upper and lower bound } \\
\text { values and places them in the } \\
\text { corresponding records nSD field }\end{array}$ & NA \\
\hline delete & remove label & Removes the label name & NA \\
\hline
\end{tabular}

\section{Dataset Parsing}

The dataset class typically contains additional meta-information within unique muscle labels that require parsing before importing to the database. These provide supplementary information about the type of experimental conditions or specific measurements within sources. This additional information is extracted using a labelparser class that uses a lookup table for all encountered labels. The labels are sorted into groups with different parsing actions. Simple groups (e.g., compartment) indicate a specific muscle compartment. Different groups required different field types to be used within the database and are described in the column Database Fields (Table 2.1). The existence of metadata (or meta label) is indicated by a Boolean field (with 'b' character attached to the label or group name). A string field (indicated with an ' $s$ ' character) contains the description of the additional meta information. Numeric label groups 'count' and 'dof' create a numeric field that contains any numeric metadata (e.g., joint angle, subject number). For example, the meta label 'COR' belongs to the 'general' group, indicating that the center of rotation method was used in the source. This creates a field bCOR=true only. Another label 'DISTAL' is in the 'compartment' group. This would create the fields bCOMPARTMENT=true and SCOMPARTMENT='DISTAL'.

The storing of meta information with labels was a necessary and convenient method to add relevant user-defined information to extracted moment arm relationships and records (i.e., entries) 
in the database. The corresponding label groups allowed parsing dataset labels by categories that could encompass countless custom labels without defining how each created meta label is handled during processing. These meta labels can be created during the digitization step and use pre-defined groups described in a standard spreadsheet (Table 2.1). Additional groups can also be created. This would require a new entry in Table 2.1 and a corresponding parsing function defined in the labelparser class. For example, a new group label 'mylabel' would need a handler function (e.g., 'label2mylabel') to be added to the class. The last parsing step is to flatten the measurements into unique datasets using all the extracted meta information in the previous step. For example, this step separates datasets collected with different methods and in different subjects.

\section{Step 3: Relational Database in MATLAB}

\section{Overview}

A relational database is an essential tool for managing moment arm relationships because of the large variation in measured morphometry and experimental conditions. The parsed datasets are imported into a relational database in MATLAB with a custom import function. Metatables are extracted and imported alongside the numerical relationships, which are passed to the database object. The simple relational database in our study uses a set of tables (i.e., metatable) to create relationships or links between common fields (usually a unique id or name) across tables. Currently, there is not an inclusive MATLAB package that has built-in querying and does not require SQL programming experience. To create a relational database, there were two options: 1) create a standalone database class that uses existing data types or 2) create a database class that utilizes the developed metatable class (see below). We selected the second option because it closer to the true essence of relational databases, and it enables the database to be a simple composition and manager of independently linked metatables.

\section{Metatables}


The metatable class was implemented as a dynamicprops class, a specific MATLAB handle class, so that modifications to the metatable would alter the object's state without explicitly declaring a modified copy of the metatable as an output, eliminating the need for persistent or global variables. The dynamicprops behavior also allows the user to dynamically add or remove properties without needing to pre-define them in the class. This feature was used to create and remove fields like MATLAB's struct and table class. These fields can be queried (qry) using standard key-value argument pairs (e.g., "name" = "john"), also known as keyword arguments. This feature is a structured search or look-up of database entries based on their attribute values. The metatable class is transparent and accessible, and it can be treated as other standard MATLAB data types (e.g., cell, struct, table).

\section{Generic Database Class}

We developed a database class like the metatable class. It is also a subclass of dynamicprops. The database can import sets of metatables from file or metatable-like inputs in the form of structure or table MATLAB variables. Besides the addition or removal of metatables, the database class serves as a container or wrapper for a set of required and optional metatable objects. This flexible implementation does not require additional memory management; all datasets are loaded into memory. For example, 32 scanned data sources with 392 unique datasets for the human lower-limb model required about $500 \mathrm{~KB}$, which is a modest memory requirement. However, the extension of this structure beyond the described data classes may require an additional memory management algorithm for large datasets. With minimal file and memory management requirements, the current implementation of the database class behaves like a generic relational database framework that can be expanded and customized to include more data and features. 


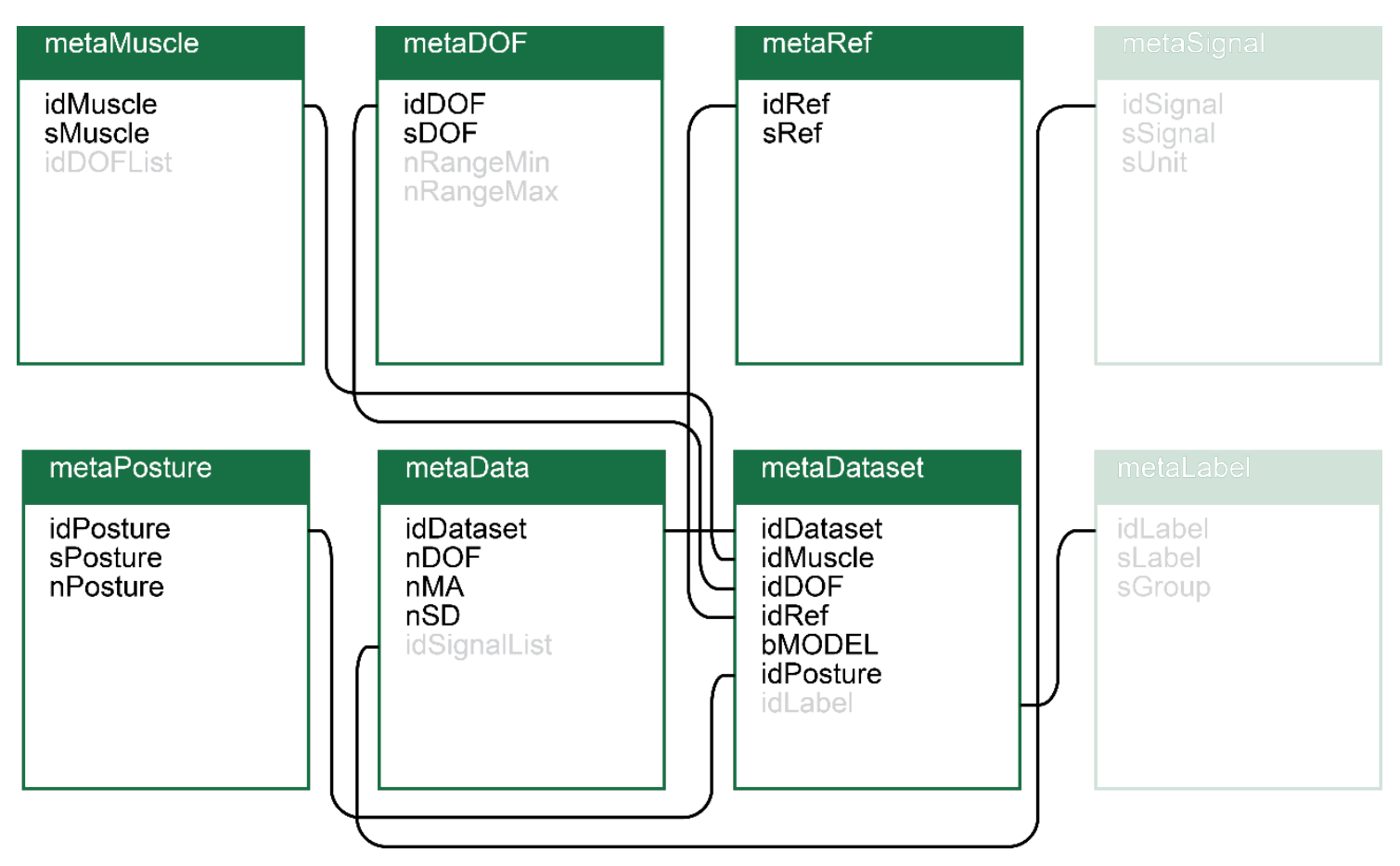

Figure 2.3. Metatables used within the relational database. The metatables shown in green are the required metatables for the database. The moment arm relationships and their meta information are stored in metaDataset and the digitized data are in metaData. The relationship between meta information is shown with a line connecting their common field(s). The fields listed in black are required and those in gray are some commonly added fields. The transparent metatables are some of the optional tables that can be used in the database.

\section{Database}

Our database is comprised of six main metatables that describe the minimal set of fields or parameters necessary to identify and parse unique datasets (see Figure 2.3). These tables capture the main attributes needed for the description of posture-dependent moment arm relationships. These relationships are expressed as records (i.e., rows) in metaDataset with the digitized data stored in metaData. The corresponding numeric data are stored in the nDOF, nMA, and nSD fields of metaData, where the attached information is linked to idDataset. The identifier field, idDataset, is used as the primary link or pointer to other stored metatables and their corresponding meta information in the database. The metatable metaDataset contains all the meta information in the primary dataset fields (e.g., idMuscle, idDOF, idRef) and the fields generated from parsing meta labels during Step 3. Information stored in these fields describe all the muscles, DOFs, sources (references), and optional user-defined attributes. These data are then retrieved with simple query commands to select appropriate dataset(s). 
[output name $]=d b .($ metatable name $) \cdot \operatorname{qry}(\{$ field name, field value,... $\},\{$ output field name\});

For example, if the user wanted to obtain the data for rectus femoris (RFEM) from (W.L. Buford et al., 1997), they would first query to find the corresponding id fields in metaMuscle and metaRef.

idMuscle $=$ db.metaMuscle.gry( $\{$ 'sMuscle', 'RFEM' $\}$, 'idMuscle');

idRef $=$ db.metaRef.gry(\{'sRef', ‘Buford1997’ $\}$, ‘idRef' $)$;

Here, we query the metatables metaMuscle and metaRef with requests to find the corresponding records (idMuscle and idRef) whose fields contain the specified values ('sMuscle' = 'RFEM' and 'sRef' = 'Buford1997'). The returned information, along with other optional meta information (e.g., idDOF), is then used to find the corresponding dataset (idDataset) to retrieve the moment arm data stored in metaData.

idDataset $=d b$. metaDataset.qry(\{'idMuscle', idMuscle, 'idRef', idRef $\}$, 'idDataset');

Data $=$ db. metaData.qry (\{'idDataset', idDataset $\},\{$ 'nDOF', 'nMA', 'nSD’ $\})$;

Here, the requested moment arms are returned as a struct with fields (nDOF, nMA, nSD) containing numeric arrays of double values. These values can be obtained independently (i.e., query each output field separately) or together (shown above) depending on preference. The relationships between limb posture (Data.nDOF) and moment arm value (Data.nMA) can then be plotted (see Figures 2.5 and 2.6). Moment arm data can also be acquired using other available meta information contained in metaDOF, metaSignal, and metaPosture, which contain information describing the DOFs and postures the data were measured (e.g., joint configuration) or in- and out-of-plane. The relationships between these variables are further plotted and analyzed.

\section{Step 4: Standardization and Comparison}

Manual Inspection for Digitization Errors 
While publications generally follow standard biomechanical and physiological conventions, reporting methods may still vary considerably. This requires a manual inspection for various digitization (e.g., flipped moment arm signs) and parsing errors (e.g., incorrectly identifying and modifying other muscle-DOF data) after import. Digitization errors are readily identified when muscle-DOF relationships are plotted. Figure 2.4 shows an example of a common error in the DOF direction sign for the sartorius data plotted in red. Here, the disagreement in the DOF direction is identified when comparing all the extracted datasets and is easily corrected. These inconsistencies can be introduced during the digitization step and are the most common error in digitizing source moment arm data. This is due to the innate differences in sign conventions used across literature for the same DOF, e.g., hip abduction-adduction can be represented with different signs. Standardizing sign conventions for muscle-DOF relationships reduce these common errors. For the lower limb, the most common DOF direction sign conventions (from negative to positive) are: 1) hip extension to flexion, 2) hip abduction to adduction, 3) hip external rotation to internal rotation, 4) knee extension to flexion, 5) ankle plantarflexion to dorsiflexion, 6) ankle eversion to inversion, and 7) toe extension to flexion. We used these conventions for all datasets in the database.

\section{Dataset Comparison}

The naming of muscles and DOFs are also different. Although these naming conventions are often consistent between groups of researchers and collaborators, these subtle differences make it less convenient to compare different datasets describing identical muscle-DOF relationships; therefore, we adjusted the muscle names and DOFs to match the style used in most other datasets. For example, gluteus muscles are often labeled as 'GMAX' or 'GLUTMAX'. Moreover, muscles that have large/several attachment locations are commonly divided into multiple compartments, which further increases the variability in naming. This is attributed by selecting a different number of compartments to describe the muscle and naming of each compartment (e.g., anterior to posterior or proximal to distal). For these occurrences, we also renamed the 
compartments to follow most datasets, or whose data is most alike (same magnitude). To enforce consistency among the collected datasets, we adopted the DOF naming convention that reflected the DOF direction sign convention described previously. Once these differences were corrected, muscle-DOF relationships were easily compared through visualizations (see Figure 2.4) and can be used to conduct analyses or the creation of "artificial" relationships.
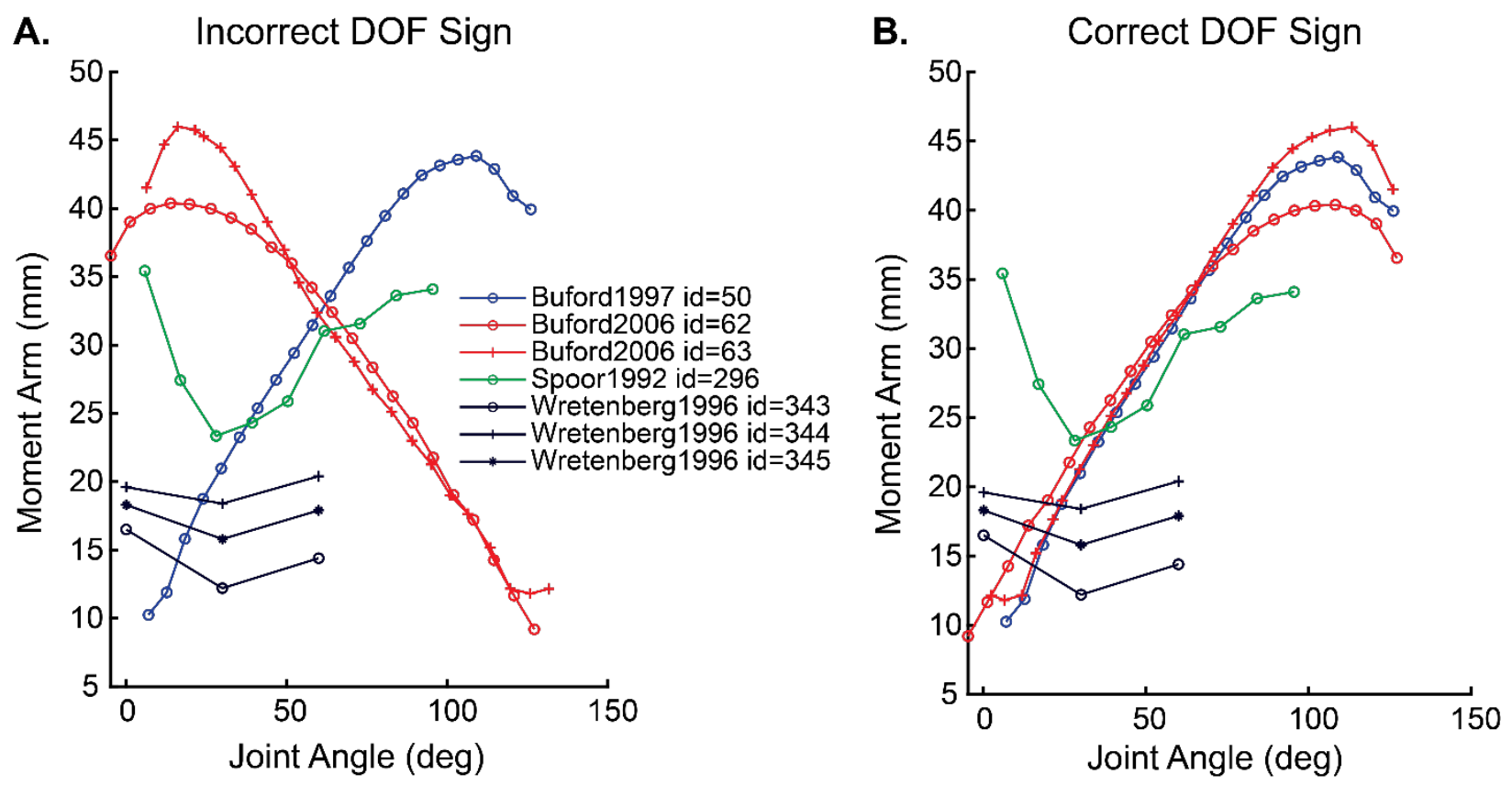

Figure 2.4. Standardizing datasets and checking for errors. A. The imported datasets for the moment arms of sartorius for the knee flexion-extension DOF. Each line color corresponds to an individual source and each marker corresponds to an individual dataset within the source. The error in the DOF direction is visible (red lines) when compared to other datasets. B. The same relationships are plotted with the appropriate correction for the imported values.

\subsection{Results}

\section{Case Study: Experimental Data for Moment Arm Validation}

Musculoskeletal models heavily rely on the data that is used to create them. Moment arm (muscleDOF relationships) datasets are often disparate and highly variable due to the inter-subject and study variabilities. With the key role these relationships have in muscle and joint torque generation capacity, the selection of these datasets is equally crucial. Here, we demonstrate the utility of our moment arm relational database to capture the accuracy and variability of measured moment 
arms by creating several comprehensive muscle-DOF relationships for select muscles spanning the ankle (achilles) and knee (rectus femoris, sartorius, semimembranosus, and semitendinosus). We collected several moment arm datasets for muscles spanning the ankle (Fath et al., 2010; Maganaris, 2004; McCullough et al., 2011; Rugg et al., 1990) and knee (Arnold et al., 2000; William L Buford \& Andersen, 2006; W.L. Buford et al., 1997; Delp et al., 1994; Hawkins, 1992; Herzog, 1992; Spoor \& van Leeuwen, 1992; Thelen et al., 2005; Visser et al., 1990; Wretenberg et al., 1996). First, we selected datasets that shared common profile characteristics, emphasizing the shape of the profile rather than magnitude (Step 1). Of the manually selected datasets, the datasets from the same source were averaged first. These datasets were averaged using spline interpolation and extrapolation for the source's entire DOF range, resampled at 5 times the original sampling rate. If these datasets did not contain the same number or location of data points, a linear interpolation was first applied for those subsets (resampled to the average number of data points) and then fitted with splines (Step 2). The resulting profiles (one for each source) were then fitted with linear extrapolation for the entire muscle-DOF range (Step 3). Linear extrapolation was used in this step to help prevent diverging points at the end ROM. The computed average for each source was combined to generate the final muscle-DOF relationship. The result was a complete muscle-DOF relationship description derived from multiple sources. All the interpolation and extrapolations were computed with MATLAB's interp1 function. A full step-by-step example for the achilles is shown in Figure 2.5 . 
A.

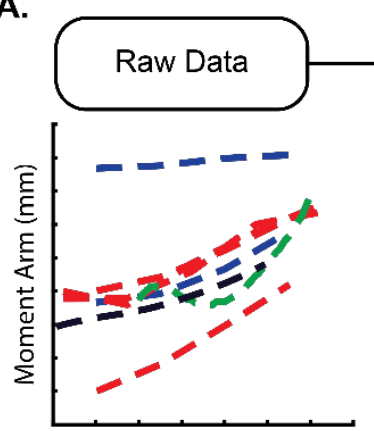

Joint Angle (deg)

B.

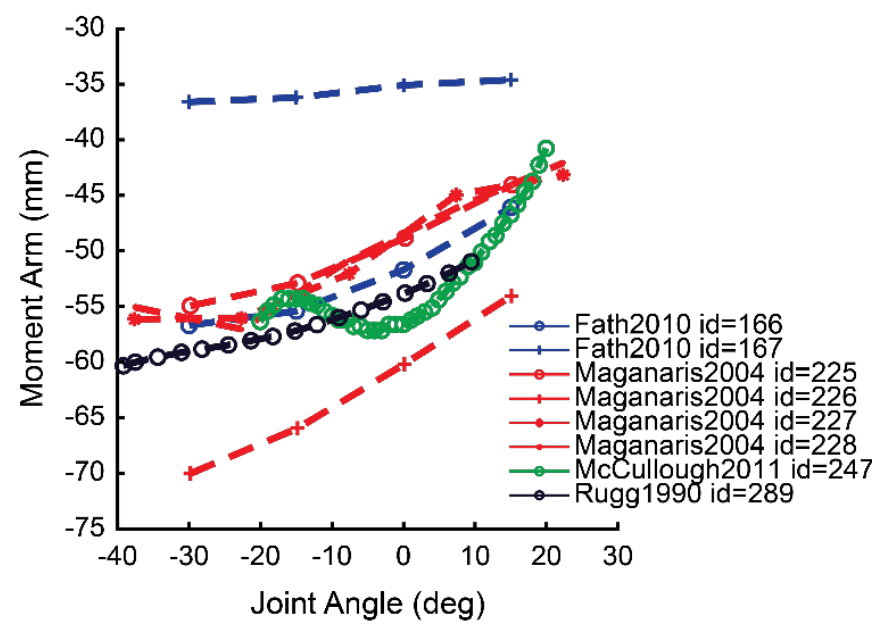

Processing $\longrightarrow$ Final

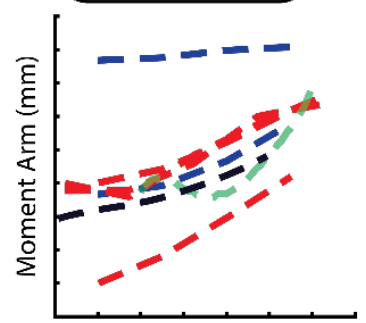

Joint Angle (deg)

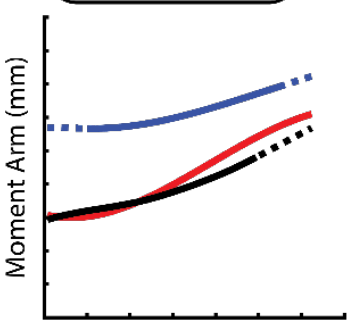

Joint Angle (deg)

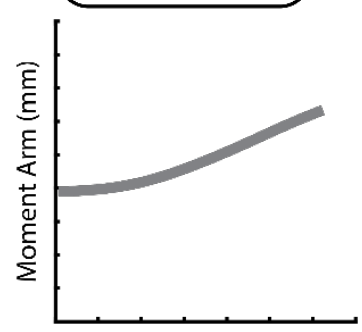

Joint Angle (deg)

C.

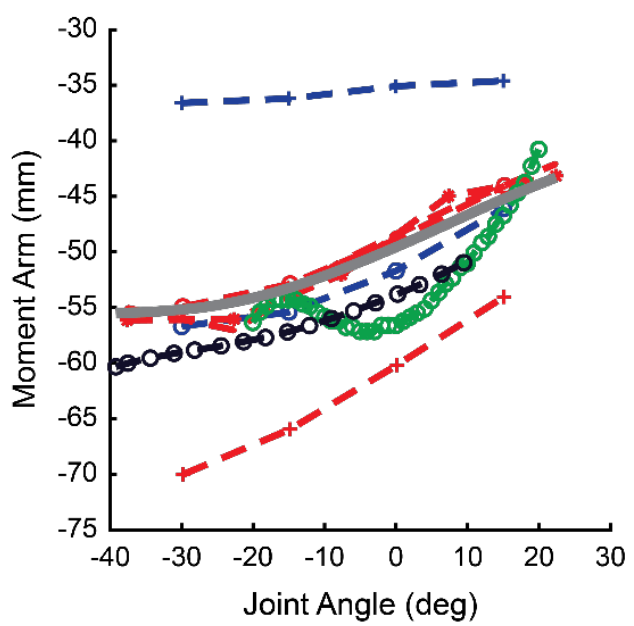

Figure 2.5 Schematic for combining muscle-DOF relationships for the achilles. A. The workflow used to combine multiple moment arm relationships for the achilles plantarflexion-dorsiflexion DOF. B. The extracted relationships from multiple sources. C. The computed standardized profile (in gray) superimposed on the extracted relationships.

\section{Achilles}

We selected to use all the collected achilles datasets for ankle plantarflexion (Fath et al., 2010; Maganaris, 2004; McCullough et al., 2011; Rugg et al., 1990) except McCullough et al. (Figure 2.5A and Figure 2.5B). This dataset was excluded because the turning points at greater plantarflexion ( $15 \mathrm{deg}$ ) and neutral (0 deg) positions would greatly affect the computed profile when extrapolating to the full ROM in Step 3. At first glance, datasets id=166 and id=266 could be neglected; however, these outlier data are a result of different measurement techniques (COR and tendon excursion) and experimental conditions (maximum voluntary contractions) within each respective study. We kept these profiles in computing our standardized profile because the other data within each study pulls the outlying data closer to reasonable differences in Step 2 
(Processing step). These data, particularly Fath et al. (Fath et al., 2010) seen in blue, were extrapolated (dashed lines) for the entire ROM before averaging to produce our standardized profile (Step 3). The final computed muscle-DOF relationship for the achilles in ankle plantarflexion is shown superimposed on all the datasets in Figure 2.5C. The standardized profile was a linearly increasing dorsiflexion moment arm that closely resembled the selected datasets. Here, we demonstrated the ability to create a comprehensive muscle-DOF relationship for the achilles from multiple datasets.

\section{Muscles at the Knee}

In addition to the achilles, we also demonstrated the ability to generate standardized muscle-DOF relationships for several muscles at the knee shown in Figure 2.6. The semitendinosus relationship was created from the data corresponding to dataset ids $19,20,24,28,52,298$, and 303 (Figure 2.6A). The omitted datasets were not selected because they did not follow the tendency of increasing moment arm with knee flexion before decreasing with larger degrees of knee flexion at the extreme ROM. The computed muscle-DOF relationship for semitendinosus represented this characteristic well (shown in gray). One of the other major hamstring muscles, semimembranosus, was also analyzed and showed considerably more variability than its superficial counterpart (Figure 2.6B); however, most of the data generally agreed in shape but differed in magnitude, which can be visually separated into two main groups shown by the blue/red lines and the green/magenta lines. These datasets were computed to produce an average profile with less amplitude than semitendinosus and separated the two distinct dataset groups. Sartorius was derived from all Buford datasets (William L Buford \& Andersen, 2006; W.L. Buford et al., 1997) shown in Figure 2.6C. One notable feature of the resultant muscle-DOF relationship is the flattened tail towards the neutral position (i.e., 0 degrees flexion). This unintended feature was produced as a result of extrapolating the slight increase in flexion moment arm seen in dataset $i d=63$. The rectus femoris was profile was constructed from all datasets except id=179 (shown in green) in Figure 2.6D. The single omitted dataset was due to the large differences in magnitude. 
The final profile (shown in gray) still reaches peak extension at the same location but contains a subtle difference in the slight deviation away from flexion towards the extreme ROM. Similar to sartorius near neutral, this also results from extrapolating the turning points at the edges of measured data.

A.

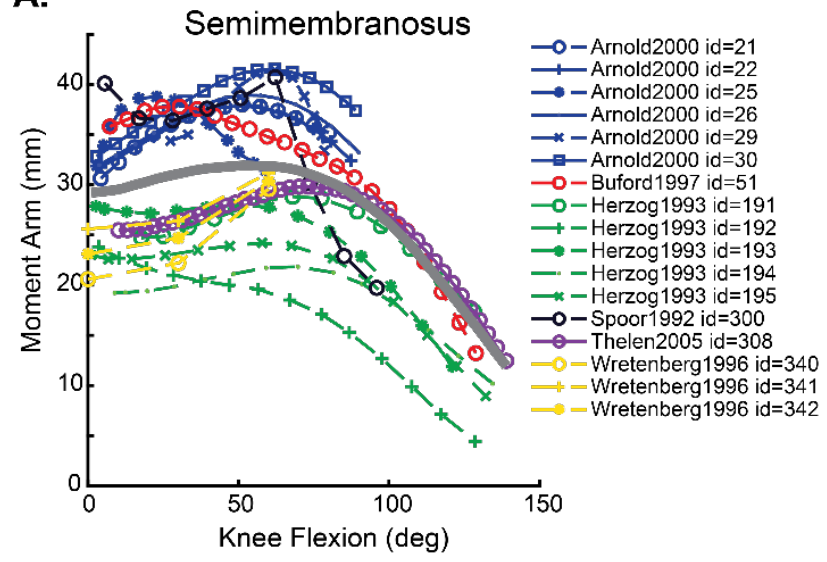

C.

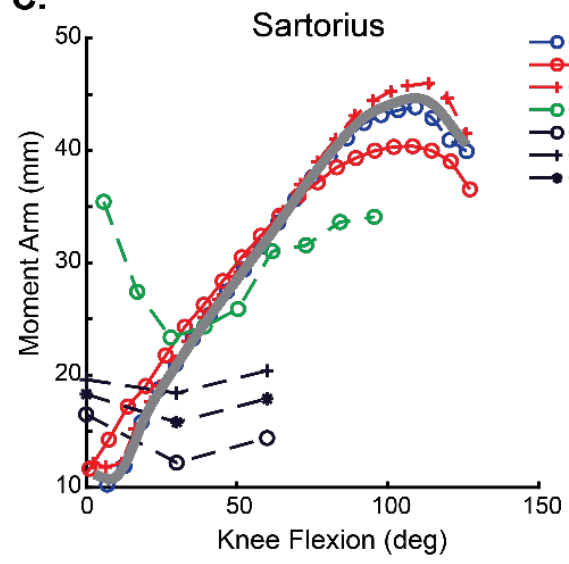

B.

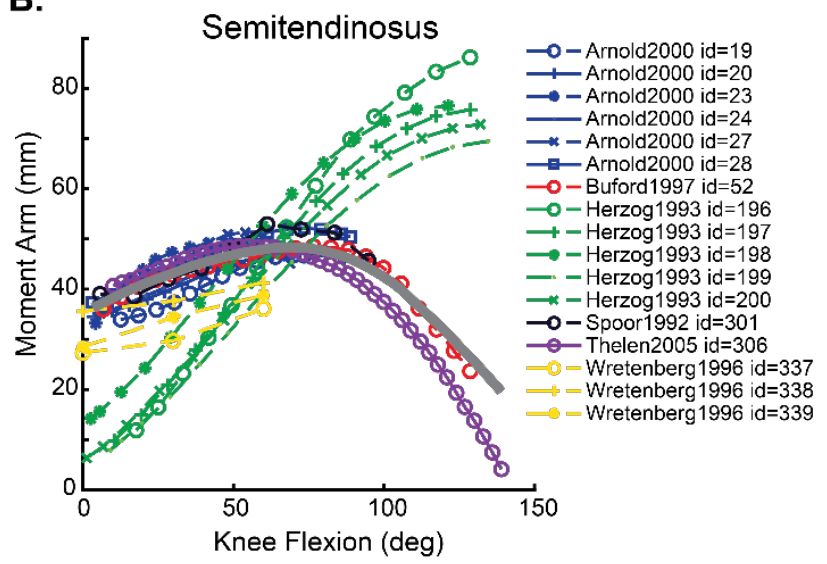

D.

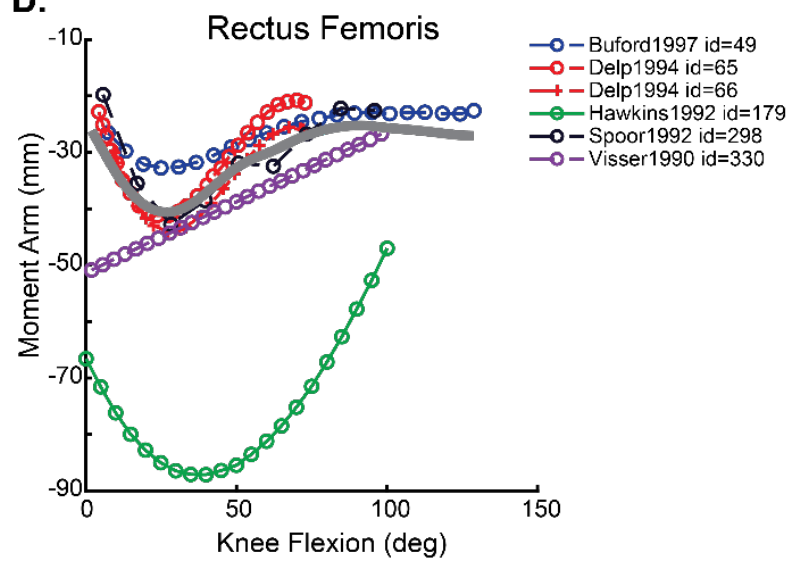

Figure 2.6. Computed standardized profiles for muscles at the knee for extension and flexion. A. Semimembranosus knee flexion moment arms. B. Semitendinosus knee flexion moment arms. C. Sartorius knee flexion moment arms. D. Rectus femoris extension moment arms.

\subsection{Discussion}

In this study, we presented an overview of a new approach for collecting and analyzing muscle moment arms to develop comprehensive muscle-DOF relationships for generic musculoskeletal models. Additionally, we developed a computational workflow and software to accompany this new approach. These developments were a composition of software tools that can easily digitize 
posture-dependent moment arm relationships, uniquely label and identify individual datasets, and create a relational database for comparison and analysis of these data. We demonstrate the use of this approach to create comprehensive moment arm profiles on a subset of muscles around the human knee and ankle. We believe our systematic workflow improves the identification and correction of errors in high-dimensional muscle moment arm profiles and will advance moment arm evaluation for validating musculoskeletal models.

This process is a needed improvement to the laborious task of collecting datasets of morphological measurements distributed across sources. The development and availability of musculoskeletal (e.g., OpenSim, SIMM, AnyBody) (Damsgaard et al., 2006; Scott L. Delp et al., 2007; Seth et al., 2018) and mechanical simulation applications (e.g., MuJoCo) (Todorov et al., 2012) provide further tools for improving the quality of biomechanical models. Our previous effort to create a similar dataset for the upper limb model took several years to complete by multiple researchers (Boots et al., 2020). By comparison, the proposed tools offer an orderly review and data management framework with improved analysis and troubleshooting that is intended for researchers with minimal programming skills. The implementation of these tools within crossplatform MATLAB application was motivated by the popular use of this tool within the scientific community and industry.

The motivation for creating a custom software pipeline for the problem of moment arm validation was a culmination of needs. First, there is an extensive list of digitization tools for extracting scanned image data (jiro, 2020; Rohatgi, 2019), as well as an abundance of open-source software for relational databasing; however, these are often separate entities and may require further development to interact with the various software to be streamlined and user-friendly. The process of collecting and analyzing human anatomical datasets would greatly benefit from integrated packages that take inputs (presumably raw data files) and process it to readily usable formats for 
analysis. Other computational biomechanics and neuroscience tools have been developed for these reasons (Mantoan et al., 2015), yet there is not such an available tool for moment arms.

Our solution was implemented in MATLAB, a popular computing software commonly used in research and industry. This platform was selected because of its ease-of-use, standardized documentation, and popularity among computational and simulation fields (Dixon et al., 2017). Although it is a tremendous tool, current solutions for a tightly integrated framework for digitization and data management does not exist. Although this point may be arguable since there are available tools for a relational database (Database Explorer, datastore) and installable digitalization toolboxes (jiro, 2020), these are not easily applicable or suitable for the discussed problem. For example, the database tools require several packages and working knowledge of a standard querying language (SQL), which is not easily accessible for scripting. Default classes struct and table are reminiscent of metatables and provide tabular structured data organized by field or variables names but do not have built-in querying. Furthermore, neither of these classes are extensible (cannot add new functionality) and force users to comply with their class limitations. With that, we developed custom MATLAB classes like struct and table to create our metatable and database tools that can be extended and have explicit querying capabilities. Regarding image digitalization software, GRABIT is a solution on the MathWorks File Exchange but is not regularly updated. This led us to select a more regulated and popular software WebPlotDigitizer (Rohatgi, 2019) for our development. Since the software is often used for online extracting and processing, most of the tools used for visualization and analysis are non-MATLAB based (e.g., JavaScript). Here, we created our custom packages to meet this need.

Despite the benefits of using the demonstrated approach to collect, create, and visualize muscle moment arms using the developed tools, there exist several limitations. This includes the high coupling (i.e., dependency) between the designed MATLAB classes. Although this allows increased coherency between tools, it significantly decreases standalone capabilities and can 
lead to large sets of dependent libraries. In this context, this makes it more difficult to independently use specific database tools without the others. Another consideration is the user's scripting or "programming" experience - at the time of development, there was not an emphasis on constructing graphical user-interfaces to interact with these tools. Furthermore, the current implementation hides most class data and properties from the command window, perhaps making it more difficult for inexperienced users to navigate to find properties like other MATLAB class types (e.g., figure and axes). The developed metatable and database classes are particularly limiting factors for large datasets since the default datatypes and algorithms were not designed for performance but rather clarity and usability.

Future work would aim to address these specific concerns, especially towards user-interface and feature development. Some additional features would include dedicated analysis toolboxes, which could include moment arm validation frameworks (Boots et al., 2020), curve-fitting tools for multi-dimensional fitting (Sartori et al., 2012; Sobinov et al., 2019) and optimizers for muscle attachment sites and path adjustments (Carbone et al., 2012; Pellikaan et al., 2014). Likewise, expanding visualization tools would help demonstrate the high-dimensional postural dependencies of muscle geometry (e.g., muscle length and moment arm) and would allow researchers and modelers alike to better account for unobserved relationships. Moreover, developing more independent yet cohesive tools would enable users to utilize specific tools for their projects or workflows. For example, this could entail using the developed WebPlotDigitizer tool, metatable, and database tools for other applications.

\subsection{Conclusion}

We developed an approach and accompanying software to create a comprehensive collection of available published moment arms for musculoskeletal model development and validation. The described workflow highlights a standardized pipeline for the digitization and storage of muscleDOF relationships of the human lower-limb. The corresponding software enables users to easily 
and quickly create a moment arm relational database from extracted datasets with automated tools. This framework addresses several of the common development and time restrictions when developing these models, like selecting appropriate muscle-DOF relationships and creating a standardized format for comparing and storing musculoskeletal datasets. Consequently, our workflow would reduce the time and effort required by developers and promote more time to analyze and simulate various conditions and components of movement. Also, this software has reduced the number of necessary tools for similar efforts and has been implemented in the userfriendly programming software MATLAB. We hope that future studies can utilize these tools to promote the growth and expansion of musculoskeletal model simulations towards related clinical and research endeavors, and lastly, further improve computational tools and standards for these applications. 


\section{Chapter 3 Validating a Lower-Limb Musculoskeletal Model}

\subsection{Abstract}

Musculoskeletal models are quickly becoming an invaluable development for simulating human locomotion and pathological conditions. However, the accuracy and viability of these models are highly dependent on the accuracy of the data used to define model behavior. These underlying data are often acquired from experimental measurements describing relationships dependent on the limb and muscle state and their subsequent behaviors within physiological ranges of motion (ROM). Yet, the required measurements of muscle moment arms are incredibly sparse and disparate, which is partially rooted in the complexity and individual differences of the human anatomy. The difficulty of validating these models increases with the complexity of morphology and requires substantial attention. Here, we utilized a novel validation pipeline to test the quality of existing musculoskeletal models. The most comprehensive model contained 13 degrees of freedom (DOFs) and 40 musculotendon actuators. The muscle paths (i.e., attachment sites or wrapping surfaces) describing the posture-dependent relationships (i.e., muscle-DOF relationships) were simulated and compared to created profiles. We show that recent musculoskeletal models developed in OpenSim accurately capture generic moment arm relationships for a subset of the hip, knee, and ankle muscle-DOFs. We also identified several structural and functional errors in muscle path geometry and moment arm profiles, particularly for muscles with long tendons (e.g., calf muscles) at the knee and ankle joints. The reduction of these errors identified in this study can be used to vastly improve the accuracy of lower limb musculoskeletal models.

\subsection{Introduction}

Our ability to move and interact with the surrounding environment enables us to perform various tasks with low cognitive load. This is achieved through the complex interactions of our nervous 
and musculoskeletal systems, which coordinate a sequence of intricate actions to generate the necessary muscle-driven torques to move. For fine movements (e.g., independent finger movements to pick up an object) the required control grows exponentially with complexity due to high-dimensional space and relationships. This problem is described as the curse of dimensionality. The ability to model and simulate these relationships remains a challenge, whether decoding motor intent from muscle activity (via electromyography) (Boots et al., 2020), muscle activation to muscle dynamics (Millard et al., 2013; Thelen, 2003; F.E. Zajac, 1989), or describing musculoskeletal architecture and function (Boots et al., 2020; Sobinov et al., 2019). Moreover, attempts to achieve accurate and robust simulations of human neuromusculoskeletal dynamics poses a difficult challenge.

The effort to create muscle-driven musculoskeletal models that emulate the same level of accuracy and redundancy of our human physiology has spanned decades of research. Recent developments in musculoskeletal modeling have expanded our understanding of locomotion through dozens of non-invasive analyses and simulations of gait (E. M. Arnold et al., 2010; Rajagopal et al., 2016), balance (Chvatal \& Ting, 2013), and muscle coordination (A. S. Arnold et al., 2007; Steele et al., 2017; Felix E. Zajac, 1993) through the development and distribution of standardized simulation tools (Damsgaard et al., 2006; Scott L. Delp et al., 2007; Seth et al., 2018). However, the rate of acceptance and usage of these models has surpassed the rate of developed validation standards. While other disciplines have well-established protocols for developing and verifying model viability, there is no common or universal procedure for musculoskeletal models (Hicks et al., 2015). Moreover, one key contributing factor leading stems from the underlying morphological assumptions and data used to develop these models. These experimental datasets containing the structural and functional relationships necessary to fullydescribe musculoskeletal dynamics are incredibly limited in consistency and quantity, imposing a significant role in the availability and selection of experimental datasets. Thus, rigorous validation 
of internal parameters and posture-dependent relationships are crucial to producing accurate and robust simulations.

Musculoskeletal models are validated with direct and indirect methodologies (Henninger et al., 2010; Hicks et al., 2015; Lund et al., 2012). Direct validation involves the direct comparison of experimental and simulated morphological values (e.g., moment arms (A. S. Arnold et al., 2000; S.L. Delp et al., 1990)). Alternatively, indirect validation often entails comparing more external or non-invasive measured and simulated quantities like muscle activity or joint torques (Boots et al., 2020; Hamner et al., 2010; Rajagopal et al., 2016). However, this type of validation can be more susceptible to computational errors. For example, using inverse kinematics to solve joint torques to compute the muscle activations that produced the observed movement is heavily reliant on the optimization algorithms used to handle muscle and joint redundancy (Bernstein, 1967; Crowninshield, 1981; Erdemir et al., 2007; McKay \& Ting, 2012). On the other hand, direct comparisons are challenging due to the availability and sparseness of morphological datasets. Furthermore, it is difficult to account for methodological and individual differences across data sources and can lead to inherited physiological inaccuracies (Goislard De Monsabert et al., 2018). Although direct validation is the preferred method since experimentally measured relationships are used to describe and compute the simulated outputs in indirect comparisons, indirect validation remains a necessary step to examine the overall behavior and function of the model. This normally includes scaling model outputs (e.g., joint moments) with a scalar (Scovil \& Ronsky, 2006) or modifying muscle parameters (e.g., moment arms, maximum isometric force) to match measurements (Nussbaum et al., 1995).

In this study, we aimed to overcome some of these musculoskeletal modeling limitations by developing a comprehensive dataset of posture-dependent moment arm relationships and used them to structurally and functionally validate a lower-limb musculoskeletal model. The primary goal was to capture the accuracy and variability of published moment arm datasets to represent 
a generic individual and use these formed relationships to validate a musculoskeletal model using the process described by Boots et al. (Boots et al., 2020).

\subsection{Methods}

\section{Overview}

In this study, we collected available published moment arm datasets from the scientific literature to create a high-quality comprehensive dataset used to validate a human lower-limb musculoskeletal model. The created validation dataset is used to validate a lower-limb model using the steps outlined by Boots et al. (Boots et al., 2020): 1) structural validation of moment arms for observed postures, 2) structural validation of moments for unobserved postures, and 3) functional validation.

\section{Model}

We elected to use a lower limb model developed by Lai et al. (Lai et al., 2017) in OpenSim (Scott L. Delp et al., 2007; Seth et al., 2018) as our baseline for validating a generic lower-limb musculoskeletal model. This model was selected because it extends the Rajagopal et al. model (Rajagopal et al., 2016) and is publicly available on OpenSim's website. The Rajagopal et al. model is one, if not the first, OpenSim musculoskeletal model to utilize recent morphological datasets containing comprehensive muscle architecture and muscle volumes of young, healthy individuals not present in previous models (E. M. Arnold et al., 2010; Carbone et al., 2015; S.L. Delp et al., 1990; Hamner et al., 2010; Horsman, Klein et al., 2007). Moreover, their model emphasized computational speed in muscle-driven simulations, which was achieved by replacing ellipsoidal wrapping surfaces with cylindrical wrappers (Rajagopal et al., 2016). Lai et al. then expanded the ROM of the model to include high hip and knee flexion-extension angles to simulate extreme postural movements such as bicycle pedaling. Additionally, the new model adjusted problematic muscle paths and parameters to reduce excessive passive forces and torques. 
The lower limb model validated in this study included some initial modifications to the Lai et al. model (Lai et al., 2017). First, since the goal of the study was to structurally and functionally validate moment arm relationships of the lower limb, we reduced the original model to only include the pelvis and the right lower limb. The resulting model contained 13 DOFs actuated by 40 massless Hill-type musculotendon units representing 31 muscles of the lower limb. Each musculotendon is modeled as a Hill-type actuator with properties described by (Millard et al., 2013; F.E. Zajac, 1989). Specific details of the normalized muscle force-length, force-velocity and tendon tension-length curves, anatomical datasets, and altered model parameters can be found in the original studies (Lai et al., 2017; Rajagopal et al., 2016). By default, the metatarsophalangeal joints were locked - these were unlocked to check for muscle path discontinuities throughout the ROM and qualitatively assess moment arm profiles since we did not identify datasets for these specific muscle-DOFs. Lastly, muscle and DOF (i.e., coordinate names) names were adjusted to be self-consistent within the model. This enabled us to utilize standardized naming conventions to compare muscle-DOF relationships across previously published OpenSim models (see Simulated Datasets). Muscle compartments were identified using numeric values (e.g., gmax1 corresponds to gluteus maximus' anterior compartment) and DOFs were renamed to use the following convention: 1) joint name, 2) positive DOF direction, and 3) limb side, all separated with underscores (e.g., hip_inrot_r corresponds with right hip adduction).

\section{Moment Arm Datasets}

\section{Overview}

The moment arm dataset used to validate our model was created from a collection of previously published and simulated data. We identified 39 available publications containing lower limb moment arm values and digitized them before importing them into a database. These values describe the posture-dependent muscle length and moment arm relationships (deemed muscleDOF relationships) for commonly observed postures in the lower limb; however, these data are 
sparse and do not contain information about unobserved postures. Furthermore, experimentally measured moment arms suffer from inherent variability due to methodological and inter-subject differences. To represent a generic population, we utilized both measured and simulated data to create a validation dataset aimed to capture the accuracy and variability observed across literature and individuals while maintaining a high-quality comprehensive dataset containing the muscle-DOF relationships necessary to fully-describe the human lower limb.

\section{Experimental Datasets}

The published moment arm data were collected, digitized, and compared to simulated moment arm values (see Simulated Models). We identified 39 published moment arm sources from our literature search that were concatenated to compose our experimental moment arm dataset (shown in Table 3.1). An initial selection procedure was implemented in an attempt to reduce the potential discrepancies and nonlinearities associated with combining multiple observations (Goislard De Monsabert et al., 2018) that are often attributed to methodological and inter-subject differences. The initial criteria eliminated literature sources whose muscle-DOF relationships were not measured as a function of the DOF angle (i.e., posture), reported less than 3 data points, or did not record values through most of the muscle's ROM. Other encountered reasons for omitting a specific source were poor dataset quality (i.e., pixelated figures or indistinguishable profiles) or questionable experimental methodology/setup. A special case was the Maganaris et al. sources, in which 4 of the 6 identified source data could be obtained in one of the other 2 sources (C. N. Maganaris, 2001; Constantinos N. Maganaris, 2004). Of the identified sources listed in Table 3.1, our initial selection criteria eliminated 7 sources, resulting in 32 sources' muscle-DOF relationships being digitized and stored in a database.

Table 3.1. Collected published moment arm dataset meta information.

\begin{tabular}{lllll}
\hline Source & Method & Measured In & \# Subjects & Subject Age \\
\hline (A. S. Arnold et al., 2000) & TE & cadaver & 3 \\
(A. S. Arnold \& Delp, 2001) & TE & cadaver & 3 \\
& & 40 &
\end{tabular}




\begin{tabular}{|c|c|c|c|c|}
\hline (Blemker \& Delp, 2005) & TE & MRI & $1(1 \mathrm{~F})$ & 27 \\
\hline (W.L. Buford et al., 1997) & TE & cadaver & $15(14 \mathrm{M}, 1 \mathrm{~F})$ & $54.8(8.2), 69(0)$ \\
\hline (William L Buford et al., 2001) & TE & cadaver & $17(15 \mathrm{M}, 2 \mathrm{~F})$ & $55.3(7.7), 69(0)$ \\
\hline $\begin{array}{l}\text { (William L Buford \& } \\
\text { Andersen, 2006) }\end{array}$ & TE & cadaver & $1(1 \mathrm{M})$ & 28 \\
\hline (Scott L. Delp et al., 1994) & TE & cadaver & $2(1 \mathrm{M}, 1 \mathrm{~F})$ & 82,90 \\
\hline (Scott L Delp et al., 1999) & TE & cadaver & 4 & \\
\hline (Dostal et al., 1986) & COR & cadaver & $1(1 \mathrm{M})$ & \\
\hline (Draganich et al., 1987) & direct load & cadaver & 6 & \\
\hline (Eng et al., 2015) & TE & cadaver & $3(2 \mathrm{M}, 1 \mathrm{~F})$ & $78(6)$ \\
\hline (Fath et al., 2010) & COR and TE & MRI & $9(7 \mathrm{M}, 2 \mathrm{~F})$ & $31(5)$ \\
\hline (Grood et al., 1984) & direct load & cadaver & $5(3 \mathrm{M}, 2 \mathrm{~F})$ & $56.7(10.8)$ \\
\hline (Hawkins, 1992) & COR & model & 1 & \\
\hline (Walter Herzog, 1992) & COR & cadaver & $5(2 M, 3 F)$ & $79.2(78-82)$ \\
\hline (Ito et al., 2000) & TE & ultrasound & $7(7 \mathrm{M})$ & $27(2)$ \\
\hline (Klein et al., 1996) & TE & cadaver & 10 & over 60 \\
\hline (Leardini \& O'Connor, 2002) & COR & model & & \\
\hline (Lee \& Piazza, 2008) & TE & ultrasound & $10(5 \mathrm{M}, 5 \mathrm{~F})$ & $21-32$ \\
\hline (Lengsfeld et al., 1997)* $^{\star}$ & direct load & cadaver & 2 & \\
\hline $\begin{array}{l}\text { (Constantinos N. Maganaris } \\
\text { et al., 1998)* }\end{array}$ & COR & MRI & $6(6 M)$ & $28(4)$ \\
\hline $\begin{array}{l}\text { (Constantinos N Maganaris } \\
\text { et al., 1999)* }\end{array}$ & COR & MRI & $6(6 M)$ & $28(4)$ \\
\hline $\begin{array}{l}\text { (Constantinos N. Maganaris } \\
\text { et al., 2000)* }\end{array}$ & TE & MRI & $6(6 M)$ & $28(4)$ \\
\hline $\begin{array}{l}(\text { Constantinos N Maganaris, } \\
2000)^{\star}\end{array}$ & TE & MRI & $6(6 M)$ & $28(4)$ \\
\hline (C. N. Maganaris, 2001) & COR & MRI & $6(6 M)$ & $24-32$ \\
\hline $\begin{array}{l}\text { (Constantinos N. Maganaris, } \\
\text { 2004) }\end{array}$ & COR and TE & MRI & $6(6 M)$ & $28(4)$ \\
\hline (McCullough et al., 2011) & TE & cadaver & $5(5 M)$ & $82(69-92)$ \\
\hline (Nemeth \& Ohlsen, 1985) & COR & CT scans & $20(10 M, 10 F)$ & $78(1), 82(4)$ \\
\hline (Nemeth \& Ohlsen, 1989) & COR & CT scans & 20 (10M, 10F) & $70(3), 63(7)$ \\
\hline (Piazza et al., 2001) & TE & cadaver & $7(2 \mathrm{M}, 5 \mathrm{~F})$ & $41-89$ \\
\hline (Rugg et al., 1990) & COR & MRI & $10(10 M)$ & $30.2(5.9)$ \\
\hline (Scheys et al., 2011)* & & model & $1(1 \mathrm{M})$ & 25 \\
\hline (Spoor et al., 1990)* & TE & cadaver & $2(2 \mathrm{M})$ & $82(0)$ \\
\hline (Spoor \& van Leeuwen, 1992) & COR & cadaver & $1(1 \mathrm{~F})$ & 89 \\
\hline (Thelen et al., 2005) & & model & 14 & $16-31$ \\
\hline
\end{tabular}


(Vaarbakken et al., 2015) T

(Visser et al., 1990)

(Wretenberg et al., 1996)

(Yamaguchi \& Zajac, 1989)
TE

TE

COR

direct load cadaver

cadaver

MRI

model
$3(2 \mathrm{M}, 1 \mathrm{~F})$

$6(2 \mathrm{M}, 3 \mathrm{~F})$

17 (10M, 7F)

1

* Source were not digitized during the initial selection criteria. () indicates the standard deviation or range. $\mathrm{M}$ indicates a male subject, $\mathrm{F}$ indicates a female subject, COR indicates the center of rotation method, TE indicates the tendon excursion method, and MRI indicates magnetic resonance imaging. Empty entries indicate unavailable or undetermined information.

\section{Validation Dataset}

The published moment arm data stored in the database were used to create moment arm relationships as the gold standard for validating a generic lower limb musculoskeletal model. Standardized naming of muscles and DOFs were required to make intra- and inter-source comparisons before selecting appropriate datasets for each muscle-DOF represented in the described model (see Model). For our digitized data, this mostly consisted of converting larger muscles' data to corresponding compartments (Scott L Delp et al., 1999; Dostal et al., 1986; Nemeth \& Ohlsen, 1985, 1989) or renaming muscles, e.g., ISO to ILIACUS in (Blemker \& Delp, 2005).

There were several assumptions when selecting and/or creating the moment arm relationships. One modification was to include the moment arm values for all QUADS datasets (Draganich et al., 1987; Grood et al., 1984; Visser et al., 1990; Yamaguchi \& Zajac, 1989) when averaging individual quadriceps muscles (e.g., rectus femoris and vastus muscles). Since all the quadriceps possess nearly identical relationships during knee flexion-extension, we believe it was reasonable to lump these unidentified datasets with each of the quad muscles. Likewise, ankle flexionextension muscle-DOF relationships typically do not explicitly measure the calf muscles (e.g., gastrocnemius and soleus). However, these powerful extensors attach to the long, elastic achilles tendon, which is well documented. We used moment arm values from achilles datasets to quantify calf plantarflexion (i.e., extension) moment arms. Source data that did not specify a specific 
muscle head or compartment that could not be identified were omitted (e.g., BIFLONG datasets (which generally agreed with the collected biceps femoris short and long head datasets)). This was particularly an issue for the gluteus muscles due to the size and compartmentalization of these muscles. Additionally, moment arms that were measured in terms of a secondary DOF (e.g., hip flexion moment arm as a function of hip rotation angle) were not used. This was due to inadequate visualization and handling of these postures (see Validation). The remaining relationships were then combined to compare against simulated moment arm profiles.

\section{Simulated Datasets}

All the muscle length and moment arm relationships were acquired through the OpenSim model with a 9-point uniform grid for the full physiological ROM of each muscle-DOF. These data were used to create "auto-generated" polynomial approximations (Sobinov et al., 2019) of the highdimensional, posture-dependent muscle length and moment arm relationships. The produced approximations generated $9^{d}$ postures per muscle, where $d$ is the number of DOFs the muscle spans. For the human lower limb, the average muscle spans 2 DOFs, creating $9^{d}(2 d+1)$ muscle values (e.g., $d^{\star} 9^{d}$ moment arm values and $9^{d}$ muscle lengths for $d^{\star} 9^{d}$ postures) or 405 values describing the average muscle $(d=2)$. Even for the average muscle spanning 2 DOFs, these measurements are extremely limited since most moment arm values are reported as a function of one DOF, not multiple. For lower limb datasets, the most commonly manipulated DOFs are in the sagittal plane, often corresponding to flexion-extension. Although this is the primary plane of motion for lower limb locomotion, modeling muscles at the hip that span 3 DOF on average and some select muscles which span 4 DOFs (e.g., tensor fascia latae) become exponentially worse to describe (5,103 and 59,049 measurements, respectively).

\section{Simulated Models}

In addition to collecting published moment arm values, we also gathered previously developed OpenSim models for additional comparison. These models are all available for download on OpenSim's website. These models were used to qualitatively compare muscle-DOF relationships 
that initially failed during moment arm validation (see Validation) and allowed us to determine whether they failed due to the reliability or quality of validation data or if the modeled relationship was incorrect. The simulated moment arms in these comparisons were computed using OpenSim's API. The model files and associated metadata were converted in metatables and stored in a separate database from moment arm datasets.

\section{Validation}

Overview

We implemented a validation process to address the structural and functional errors and limitations commonly seen in musculoskeletal modeling: simulated muscle path errors in experimentally 1) observed postures and 2) unobserved postures. To overcome these issues, we evaluated our model with a validation framework that verifies accurate representations of musculoskeletal dynamics by identifying the importance of muscle architecture and subsequent moment arm relationships (Boots et al., 2020). Here, the following sections will summarize their validation procedure.

\section{Step 1: Validation of experimentally observed postures}

The anatomical integrity of each musculotendon's geometry was evaluated by comparing the validation dataset's muscle-DOF relationships to model simulations (see Simulated Datasets). The quality of the simulated relationships was qualitatively and quantitatively assessed for profile errors and to estimate 'good enough' approximations. The quantitative metrics used to determine 'good enough' quality were either 1 ) the root mean square error (RMSE) $<1 \mathrm{~mm}$ and the Pearson correlation coefficient $(\mathrm{R})>0.7$; or 2 ) RMSE normalized to the range of validation moment arm values per DOF $<0.4$ and $R>0.7$. These values were chosen as the "operational definition of acceptable quality" and were based on previous models and real-time decoding performance (Boots et al., 2020). The qualitative metric categorically grouped muscle-DOF relationships into dynamic and static profile errors (see Results). 


\section{Step 2: Validation of experimentally unobserved postures}

Due to the posture-dependencies caused by muscle length and consequent geometry changes and the sparsity of moment arm data for physiological postures, validation can be a daunting and exhaustive task. Moreover, the examination of approximated high-dimensional relationships is limited and cannot guarantee valid behavior. To address the accuracy of structural and functional behavior for unobserved postures, additional tools were needed. One of these developed tools evaluated postures to find occurrences where the moment arm value crosses zero, indicating a change in muscle function (i.e. flexion to extension). All "zero-crossing" instances were examined to assess if the change in muscle function was physiologically valid. The other tool analyzed postural extrema, where typical structural errors due to poor wrapping geometry or zero-crossings occur.

\subsection{Results}

In this study, we developed generic muscle-DOF relationships by combining multiple datasets to evaluate a recently published OpenSim musculoskeletal model (Lai et al., 2017) of the lower limb using the validation process outlined in (Boots et al., 2020). Here, we utilized both qualitative and quantitative metrics to evaluate moment arms profiles. These were used to assess the model for structural errors in muscle path geometry (e.g., muscle moves through the bone) and functional errors where moment arm sign flips (i.e., zero-crossing) for both observed and unobserved postures.

The model contained 63 zero-crossing locations shown in Figure 3.1. The most occurred at the hip (55) and the least at the knee (2). Of those at the hip, 19 occurred in hip flexion, 15 in hip adduction, and 21 in hip rotation. Most of the crossing locations at the hip were in the adductor and gluteus muscles, some of which are expected since they are reflected in their published experimental datasets. Only the rectus femoris and tensor fascia latae muscles experienced crossing events at the knee. For the ankle, the peroneus and tibialis muscles were responsible 
for all zero-crossings, all except one occurring in the first half of the muscles' ROM. The distribution of zero-crossing locations showed a relatively neutral preference in the ROM.

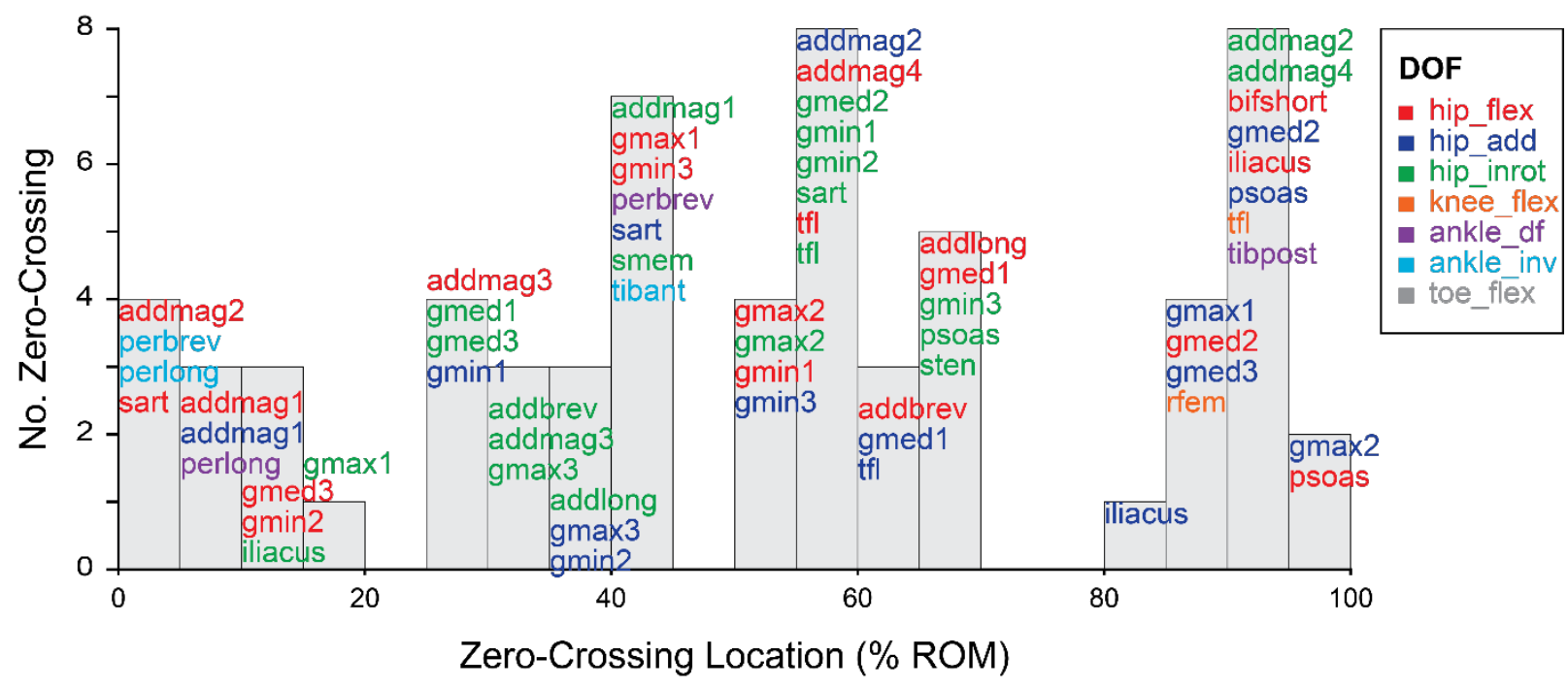

Figure 3.1. Histogram of moment arm zero-crossing locations as a function of ROM. All muscles with moment arm zero-crossings are shown for each muscle-DOF they occur in. Each color corresponds to a DOF (see legend) in the model.

The qualitative comparison of measured and simulated muscle-DOF relationships and their categorical values are shown in Figure 3.2. The 40 muscle paths representing the 33 muscles of the lower limb across 7 DOFs were assessed. Each muscle spanned 2-3 DOFs on average with the vastus muscles spanning 1 DOF and tensor fascia latae spanning 4 DOFs. Of the 96 muscleDOF relationships described, 42 were omitted due to insufficient or partial moment arm measurements (blue) (see Figure 3.2A). The categories ranged from 'good' (green) to 'bad' (red) shown in Figure 3.2B. The 'good' category indicated that the moment arm profile for the specified muscle-DOF met the evaluation criteria (see Validation). The worst category (shown in red) was given to moment arm profiles with an unvalidated zero-crossing location. The other categories (yellow, orange, and pink) indicate specific errors corresponding to scaling, dynamic, and overall profile errors. These represent magnitude (yellow), minor (orange), or significant (pink) profile disagreements between the measured and simulated profiles. 
The original model did not include any 'bad' category errors. The best muscles were semimembranosus and semitendinosus with all 'good' categories and the worst was peroneus longus with all pink categories. The average normalized RMS and R values for all profiles were $(0.65 \pm 1.0 \mathrm{SD})$ and $(0.62 \pm 0.60 \mathrm{SD})$. For muscle-DOFs in the 'good' category, these values were $(0.17 \pm 0.12 \mathrm{SD})$ and $(0.951 \pm 0.06 \mathrm{SD})$, respectively. Each category was also assigned a score scaled from 0 to 4 , with 0 corresponding to the 'bad' category and 4 corresponding with the 'good' category. The model scored 167 out of a possible 216 (assumes all 54 checked muscle-DOFs are scored with 4), an overall validation score of $77.3 \%$. The best DOF was hip rotation with $93.2 \%$ and the worst was ankle inversion with $56.3 \%$, followed closely by ankle flexion (56.8\%). 
A.

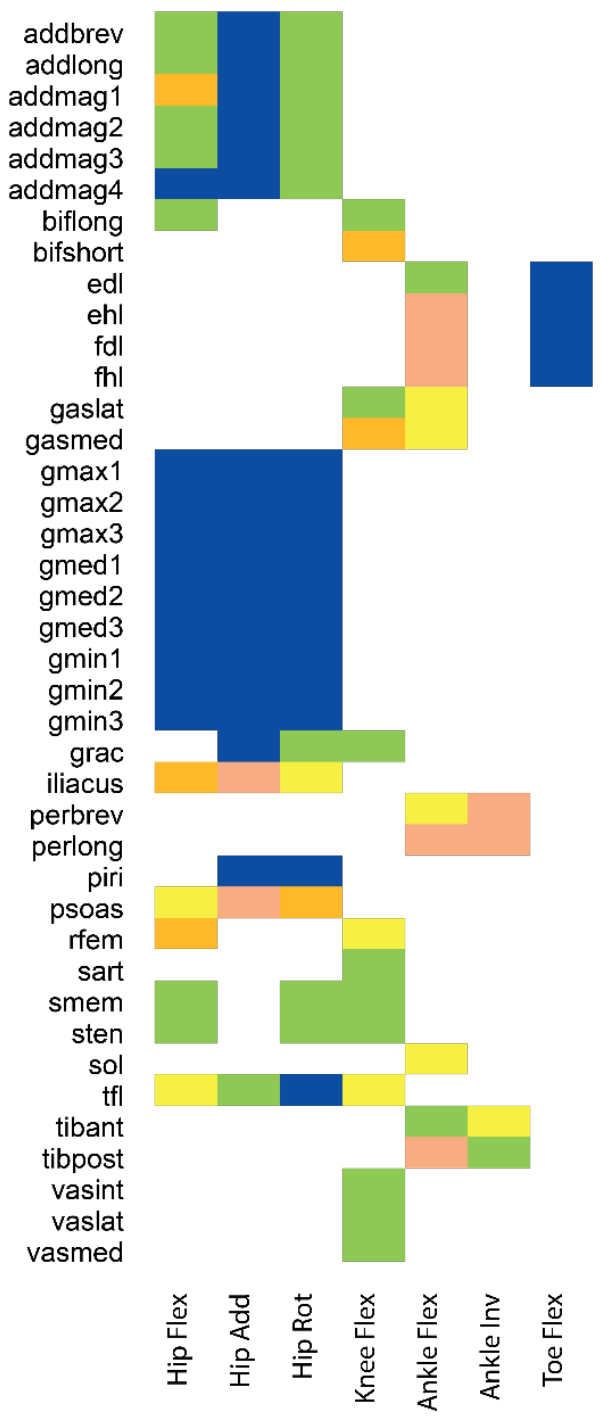

B.

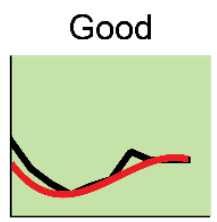

Scaling Error

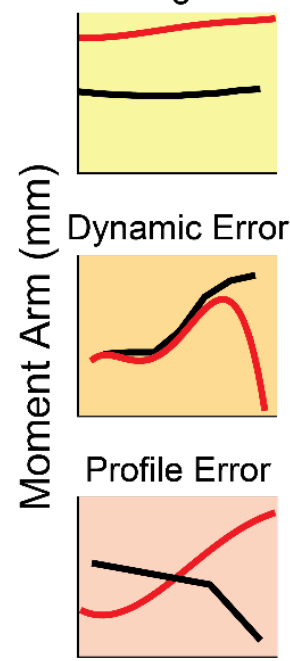

Opposite

Sign Error

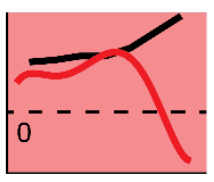

Full ROM

Figure 3.2. Categorical comparison of moment arm profiles for all muscles and DOFs. A. The original model (Lai et al., 2017) evaluated for profiles errors. The muscles are labeled vertically and the DOFs are labeled horizontally. The categorical values are shown for each modeled muscle-DOF relationship. B. Examples of the categories used to assess moment arm profile errors.

The created muscle-DOF relationships from published experimental datasets were compared to polynomial approximated and OpenSim simulated profiles (see Validation). Some of the most commonly analyzed agonist-antagonist (e.g., flexor-extensor) muscles at the knee and ankle DOFs are compared in Figure 3.3. These included the rectus femoris and semimembranosus for knee flexion, soleus and tibialis anterior for ankle flexion, and peroneus brevis and tibialis posterior for ankle inversion. The created moment arm profiles (thick black line) shown in Figure 
3.3A agree well with the measured datasets - the only exceptions are the peroneus brevis and tibialis posterior lines shown in red, which strongly disagree with the created profiles. Similarly, the simulated profile (red) in Figure 3.3B for peroneus brevis reflects the disagreement in the two measured moment datasets (Klein et al., 1996; McCullough et al., 2011) and is evaluated accordingly ('pink' category) despite the corresponding moment arm profiles in published OpenSim models (see Figure 3.3C) agreeing with the simulated profile. The rectus femoris, soleus, and tibialis posterior muscles also show the general trend of measured moment arm profiles slightly disagreeing with simulated profiles; however, these muscle-DOFs still fall within the green, yellow, and orange categories. Overall, the presented OpenSim moment arm profiles in Figure 3.3C agree amongst each other but with some containing a noticeable difference in scaling. These could be generally categorized into two groups: one group consisting of the Arnold, Lai, and Rajagopal models and the second group consisting of the Gait2392, Hamner, and Shelburne models. One common feature across all simulated profiles was their more pronounced parabolic shape compared to measured datasets. This was less evident in the rectus femoris, which all OpenSim models included at least one cusp at extreme positions in knee flexion (around 0 and 100 degrees). 
A.
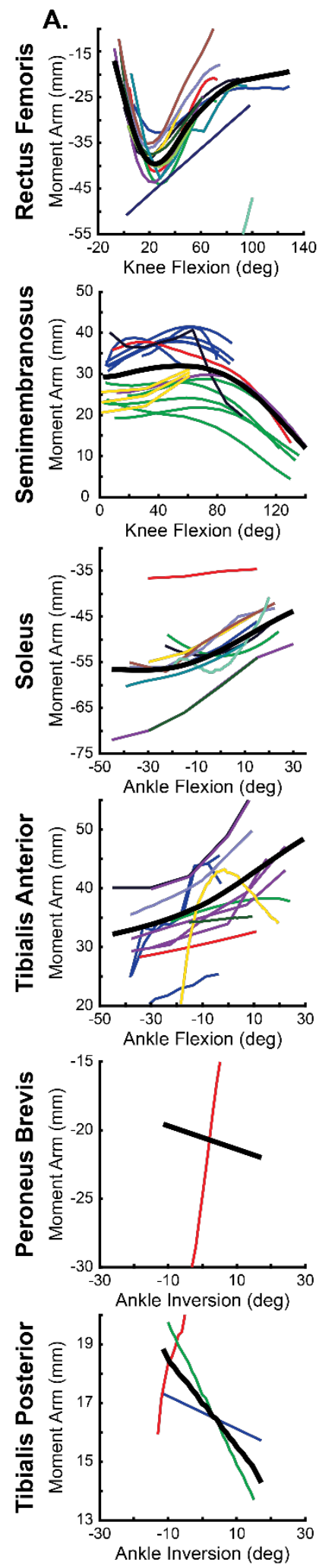

B.
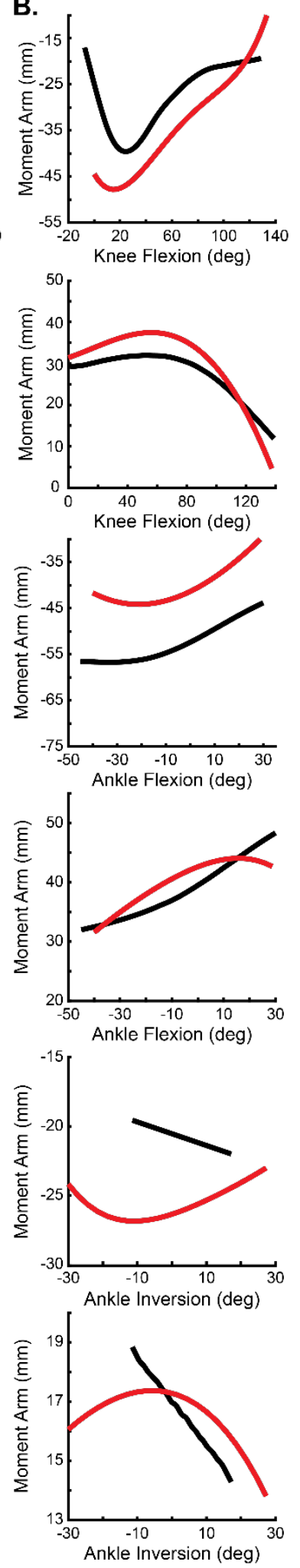

C.
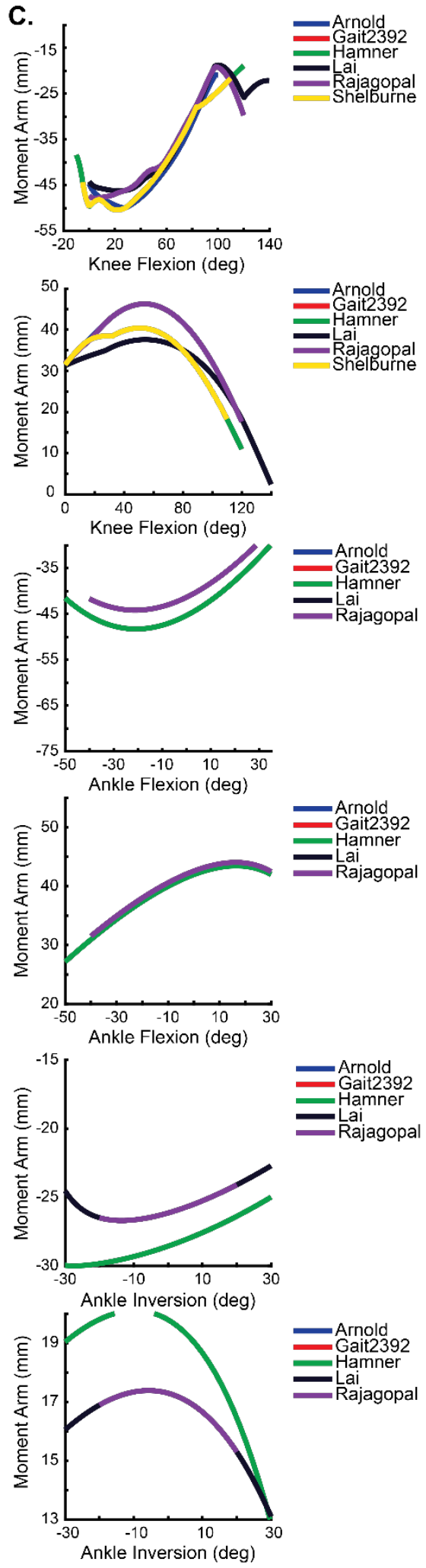

Figure 3.3. Comparison of measured and simulated moment arms for knee and ankle muscles. A. All available measured moment arm data and created moment arm profile from combining data. B. Comparison of created and simulated moment arms in the validation process. C. Simulated moment arm profiles for multiple OpenSim models. 
The muscle paths were also examined for structural errors for a range of postures. Here, we moved each joint through the entire ROM in different postures to check for incorrect muscle geometry that causes the muscle to go through bone or jump around/through wrapping surfaces. We identified several of these occurrences in the model and show a subset of these in Figure 3.4. These locations mainly occur in potentially unphysiological or postures outside of gait (e.g., internally rotated hip in hyperflexion); however, notable errors in single-plane movements for the gluteus medius and minimus, sartoris, quadriceps, and several muscles at the ankle (e.g., peroneus and extensor digitorum/hallucis muscles) were identified and are shown below. For the gluteus muscles, the muscles paths move through the ilium and pelvic bones of the pelvis in hip flexion-extension and abduction-adduction movements (Figure 3.4A). The rectus femoris and sartorius muscle move through the head/neck of the femur during high hip extension (Figure 3.4B) and all the quadriceps move through the distal portion of the femur (Figure 3.4C) in knee flexion. Some of the problematic muscles at the foot (Figure 3.4D) are defined moving through bone in the neutral position (i.e., 0 deg). These are further exaggerated during ankle flexion-extension and eversion-inversion movements. 
A.

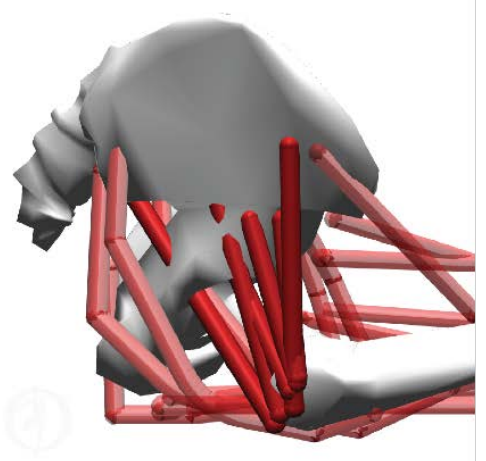

C.

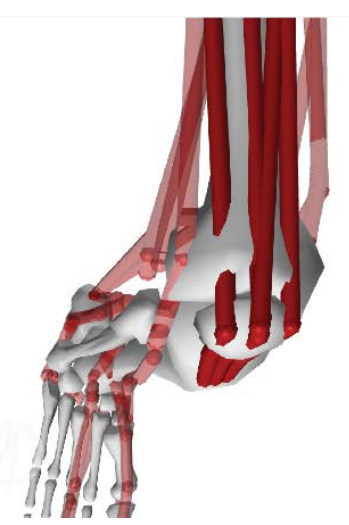

B.

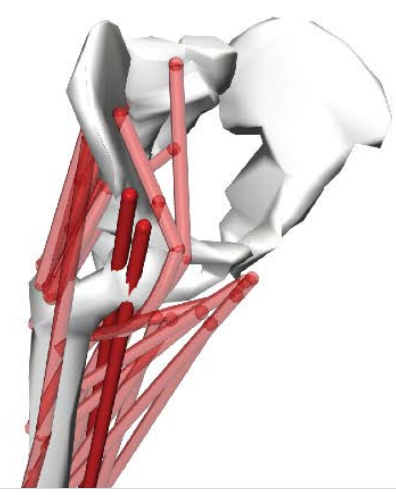

D.

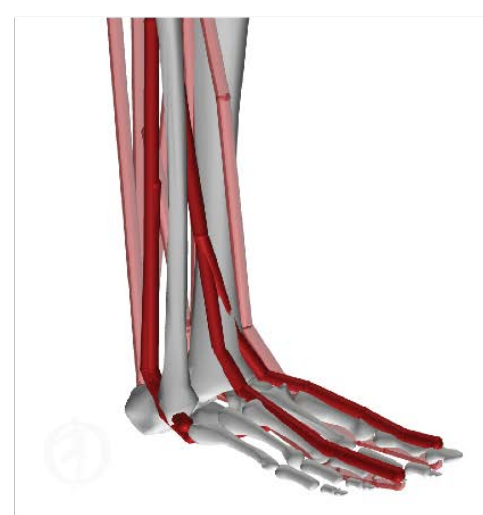

Figure 3.4. Identified muscle path error examples at each joint. The problematic muscle paths are highlighted in red and surrounding muscle paths are opaque. A. The gluteus medius and minimus muscle compartments at 80 degrees hip flexion going through the pelvis. B. The rectus femoris and sartorius muscles at 30 degrees hip extension at the femur. C. The quadriceps (rectus femoris, vastus intermedius, vastus lateralis, and vastus medius) for high knee flexion (90 degrees shown). D. Peroneus (2 left-most) and extensor digitorium/hallicus (2 right-most) muscles in the neutral position at the ankle joint.

\subsection{Discussion}

We evaluated a published lower-limb musculoskeletal model developed in OpenSim using a structural validation process that checks for muscle path and moment arm errors. In this process, we first analyzed moment arm profiles by comparing the created muscle-DOF relationships from measured datasets to simulated moment arms (see Datasets). The qualitative profile values shown in Figure 3.2 demonstrate that the model compares well in the hip flexion, hip rotation, and knee flexion DOFs. These muscle-DOFs relationships score highly due to a large number of highquality and consistent moment arm measurements from published sources. Likewise, previous OpenSim models have emphasized these specific relationships to simulate gait, running, and 
pedaling movements (Hamner et al., 2010; Lai et al., 2017; Rajagopal et al., 2016). This was also reflected in the similarity of simulated profiles compared to measurements shown in Figure 3.3. Moreover, this is partially attributed to more recent models qualitatively comparing these simulated profiles to select measured datasets (A. S. Arnold et al., 2000; E. M. Arnold et al., 2010; Lai et al., 2017; Rajagopal et al., 2016). However, the ankle flexion-extension DOF was the worst in our analysis.

The strongest extensors (gastrocnemius and soleus muscles) and flexors of the ankle (tibialis anterior) were generally in agreement with moment arm measurements and previous models (see Figure 3.3). These muscle-DOFs also included several high-quality datasets. The ankle extensors underestimated simulated moment arms. These errors can be attributed to selecting achilles measurements, a long tendon connecting the calves to the heel bone, to describe the calf muscles' moment arm relationships. This would possibly suggest that ankle joint torques would be underestimated but these torques are often grossly overestimated due to the passive component of the muscle force-length relationship that accounts for the tendon (Millard et al., 2013; F.E. Zajac, 1989). Modifications to parameters to counteract these errors often involve increasing tendon slack length and decreasing optimal fiber length (Lai et al., 2017), effectively resulting in a shorter muscle that operates more on the ascending (increasing) portion of the forcelength curve where the passive component is less significant. This is commonly observed in distally located muscles because they rely on longer, elastic tendons to translate muscle length changes due to contractions to actuate the joint (Yamaguchi, 2001). Furthermore, inclusion/exclusion of an additional elastic element modeling tendon in the internal muscle model would also drastically alter these dynamics based on its stiffness (i.e., rigid tendon assumption). The extent of these changes may not be fully realized without the comparisons conducted in this study. Without acknowledging the muscle geometry component of torque production, these parameters cannot be appropriately scaled to reduce observed errors. 
Other muscle-DOF relationships at the ankle for the peroneus, extensor digitorium/hallicus, and flexor digitorium/hallicus muscles were deemed unsatisfactory in the validation process. Initially, these comparisons were significantly inaccurate (see peroneus brevis in Figure 3.3). Yet, all the simulated relationships from previously published models relatively agree with those simulated during validation. This raised several questions about the actual quality of these failed relationships. Upon inspection, it was evident that the simulated profiles received poor evaluations due to inconsistencies in measured datasets. It was also apparent these muscle-DOFs also contained fewer total datasets for selection. This example presents an interesting scenario in model validation where the modeler, or domain expert, must decide which relationships are appropriate for the developed model. In this context, we would assume confidence in muscleDOF relationships with largely consistent measurements, and that muscles with similar function would share common characteristics (Boots et al., 2020; Lieber, 2000; Sobinov, 2019). This could result in selecting the simulated relationships to be the ground truth despite having measurements like those shown for muscles in ankle eversion-inversion (Figure 3.3). The profiles for the tibialis posterior also pose a similar question in data viability and confidence for individual datasets, where measured datasets whose overall characteristics and corresponding simulated data evaluate as 'good' but demonstrate different profiles. There were some instances where multiple and consistent datasets were used to validate a simulated moment arm profile but resultant in large disagreements; however, a single or minority of other measurements for the muscle-DOF would strongly agree with the simulated profile. This could indicate 1) the models may better reflect subject-specific moment arm characteristics corresponding to minority datasets or 2) the majority of measurements are inaccurate. The later might be a result of source variability. These observations highlight the importance of qualitatively and quantitatively evaluating simulated relationships against previously published experimental measurements and model simulations (Henninger et al., 2010; Hicks et al., 2015; Lund et al., 2012). 
The muscle paths for all musculotendons were functionally validated by checking for moment arm zero-crossing locations in all possible postures for each DOF's full ROM (see Figure 3.1). The hip muscles were especially susceptible to zero-crossings, particularly for the adductor and gluteus muscles. Since the gluteus muscles were not analyzed in this study, we were not able to determine if the identified locations were physiologically valid, which would be indicated in measured moment arm profiles. Likewise, the hip adduction determinations were also not feasible; still, we expected a higher number of zero-crossings at the hip joint than at other joints because select muscle-DOFs around the hip change function in certain postures, and are in general, more complex. This is due to simpler muscles spanning the knee and ankle only actuating 1 to 2 DOFs on average as opposed to 3 to 4 DOFs at the hip. There also exist more possible postural combinations due to larger ROM and more DOFs. Both facts are eluded by the number of required samples necessary to describe these relationships. The number of crossing locations can also provide valuable insight into which muscles or DOFs are more susceptible to structural errors in muscle path geometry for these reasons.

We evaluated the model's muscle paths for the entire ROM for structural inaccuracies (e.g., muscle through bone and/or point "jumps" from poor wrapping surfaces). Here, we discovered similar results to those in the zero-crossing analysis with the hip and ankle being the worst DOFs for these types of errors. Although most were in non-physiological postures, several postures were identified in ROMs that are used to simulate various tasks, especially those with high flexionextension movements (see Figure 3.4). These could partially explain some of the determined zero-crossing locations or unsmooth/discontinuous portions observed in simulated moment arm profiles (e.g., rectus femoris in Figure 3.3). For the quadriceps, we believe the disagreement in measured and simulated profiles is due to the differences in moment arm measurement techniques (direct load and TE), with direct load datasets exhibiting the substantial changes in moment arm around peak extension. However, a common simplification of the knee mechanism 
is to extend the quads to represent the patellar tendon (similar to the calf muscles modeled lumped with the achilles) and several patellar tendon moment arm datasets (W.L. Buford et al., 1997; W Herzog \& Read, 1993) also exhibit this feature in high knee flexion and increased extension moment arm. For the ankle, problematic muscle paths seem to stem from the initial definition of path geometry which can be seen traveling through bone in the neutral position. We suspect that modifications to these paths in the neutral posture would fix most occurrences in the ankle and with additional wrapping surfaces to prevent other locations in the ROM (e.g., the peroneus muscles passing through the lateral malleolus of the fibula).

There are several limitations in this study, most relating to the measured moment arm datasets used to create and compare muscle-DOF relationships during the validation process. Specifically, the availability and sparseness of moment arm data severely hinder the ability to fully-describe the necessary muscle-DOF relationships for musculoskeletal simulations. Of those available measured datasets, there are still large inherent variations from inter-subject differences compounded on differences in measurement (e.g., cadaver, TE) and reporting methods (e.g., figures or tables). To create generic musculoskeletal models, individual anatomical variability must be appropriately expressed by combining all available data; however, these inconsistencies in published source data make it extremely difficult to 1) safely merge needed datasets and 2) capture all the posture-dependent relationships for observed and unobserved postures. Another limitation was the inability to properly evaluate all modeled muscle-DOFs. This is an extension of the primary limitation described above. However, of the models analyzed, the current model would be our recommendation for a baseline model based on the observations made in this study. Furthermore, the comparison of simulated moment arm profiles approximated with 'good enough' polynomials and those computed in OpenSim are not identical and occasionally generate minor differences in moment arm profile characteristics. Despite these limitations, we successfully 
gathered a collection of published moment arm datasets to highlight common structural and functional errors in a previous human lower-limb musculoskeletal model.

\subsection{Conclusion}

In this study, we assembled a moment arm database populated with available experimentally measured datasets to create 'generic' muscle-DOF relationships for the human lower limb. These developed relationships were used in a validation process to evaluate a current musculoskeletal model for structural and functional errors in muscle path geometry and moment arm profiles. Our results suggest that current musculoskeletal models can reliably be validated for a subset of muscle-DOF relationships spanning the hip, knee, and ankle joints with the moment arm datasets used in this study. Select DOFs are shown to meet our validation standards, indicating that this model, or similar ones, can produce accurate muscle torques based on the validated relationships in these DOFs. We also highlight problematic muscles and DOFs to address moment arm datasets that are needed to accurately model the lower limb and to guide future work in lower limb musculoskeletal development. Overall, we believe improvements in these areas will help improve the physiological accuracy and robustness of lower-limb musculoskeletal models. 


\section{Chapter 4 Discussion and Future Work}

This thesis discusses current applications and challenges in developing and validating musculoskeletal models. The background that is necessary to understand the fundamentals of musculoskeletal modeling are presented through topics relating to muscle physiology, internal models (e.g., Hill-type muscle models), muscle architecture, and validation standards within computational biomechanics and motor control fields, as well as the experimental data that accompany these concepts and models. Specifically, my research focused on developing standardized software and workflows for acquiring and managing musculoskeletal datasets (e.g., moment arms) to generate datasets that can be used to validate musculoskeletal models. In chapter 2, I outline the developed computational workflow and software tools used to extract moment arm datasets from published literature into a custom relational database to generate 'generic' moment arm profiles for developing and validating lower limb models. In chapter 3, I use the created moment arm relationships to evaluate a published musculoskeletal model for muscle path and moment arm errors with a recently developed validation framework.

\subsection{Moment Arm Datasets}

In chapter 2, we developed a new approach for collecting and managing muscle moment arm datasets for musculoskeletal modeling. The collected datasets were used to generate a comprehensive validation dataset used for creating musculoskeletal models. We also developed corresponding software and workflows that automated most of the data acquisition and database creation steps in this process. Similar efforts to create a complete dataset of all the relationships to model and validate the human lower arm and hand took several years for multiple researchers to create (Boots et al., 2020). The presented approach provides a linear and systematic step-bystep process for digitizing and storing all the necessary data (see Figure 2.1). This drastically improved current processes for collecting the hundreds of needed datasets to fully describe 
musculoskeletal relationships which heavily rely on tedious manual interventions like creating excel spreadsheets. Previous open-source tools are capable of extracting and digitizing imagestored datasets (jiro, 2020; Rohatgi, 2019) and managing relational databases; however, these are often independent tools with little coherence between them. They also often require more technical or programming skills (e.g., SQL) to properly navigate and produce desired outputs (e.g., data tables, database queries). Our solution was developed as an all-inclusive software toolbox in MATLAB (a popular computational software for engineers and researchers) that would easily allow users to retrieve datasets stored in literature figures and tables and quickly generate organized tables or databases (see Figure 2.3). Furthermore, we include additional tools that improve analysis and troubleshooting of these datasets which are focused and/or intended for individuals with no to little programming experience (see Figure 2.4). The accumulated datasets could then be used to conduct a variety of meta and numerical analysis, e.g., averaging datasets.

There are several limitations to the presented approach. The first is locating and acquiring the published literature containing the necessary moment arm relationships for lower limb muscles. Available datasets are often very disparate and sparse, which can introduce nonlinearities and physiological inaccuracies when combining datasets (Goislard De Monsabert et al., 2018) that include differences in individuals and methodologies. These were generally handled by including labels and meta information to extracted datasets during digitization and then intra- and intersource averaging with linear and spline extrapolations. However, extreme care is required to implement consistent naming across datasets and literature, which could ultimately result in inappropriate combinations or analysis of these datasets. Similarly, more sophisticated selection or combination procedures for the data could be used to improve the current implementation (e.g., unsmooth transitions between profile features) shown in Figure 2.5. Moreover, the developed software (although intuitive) lacks graphical user-interfaces for more convenient point-and-click types of commands. The primary focus was on providing a framework and scripting tools that 
could be expanded on in the future. Likewise, some of the developed MATLAB classes (e.g., metatable and database) were not initially built or designed for computational and size efficiency, which could result in unexpected or undesirable consequences for larger datasets (e.g., genome sequencing or machine learning).

\subsection{Musculoskeletal Models}

In chapter 3, we evaluated a previously published lower limb musculoskeletal model created in OpenSim (Scott L. Delp et al., 2007; Lai et al., 2017; Seth et al., 2018). The model was checked for common musculoskeletal structural and functional errors in muscle path geometry and subsequent moment arm relationships based on the work described in (Boots et al., 2020). We

used the created relationships in chapter 2 as our gold standard for comparing measured and simulated moment arm profiles, a common and often preferred validation approach (Henninger et al., 2010; Hicks et al., 2015; Lund et al., 2012). Figure 3.2 shows the qualitative scores and profile errors for these comparisons, which easily highlighted good and bad muscle-DOF relationships. For those muscle-DOFs evaluated as 'good', these relationships generally contained more measurements and consistent profiles compared to those with worse evaluations. The ankle muscle-DOFs, except for the calf and tibialis anterior muscles, were particularly poor, with both flexion-extension and eversion-inversion DOFs containing 1-2 inconsistent measured datasets on average. Yet, examining these bad profiles and comparing them to previous lower limb models (see Figure 3.3) demonstrated the need for both qualitative and quantitative metrics for direct and indirect comparisons (Hicks et al., 2015) by posing several scenarios that call into question the accuracy and validity of both the measured and simulated data. Similarly, it also helped identify specific scaling and discontinuity errors observed in muscles at the knee (quadriceps) and ankle (calf muscles) that may be due to common modeling assumptions (e.g., patellar and achilles tendons). Due to the limited and sparse measurements used to validate simulated moment arms, additional checks for muscle path geometry or moment arms errors in 
unobserved postures were necessary to locate instances of muscle traveling through bone (see Figure 3.4) or changes in muscle function (i.e., moment arm zero-crossing) shown in Figure 3.1. These observations only reinforce the need for validation tools and standards described in (Boots et al., 2020), and moreover, highlight the data and steps required to advance musculoskeletal models.

The measured datasets needed to fully-describe musculoskeletal dynamics remains the largest challenge for these models. Without adequate measurements to validate accurate physiological relationships, there can be some doubts about whether to trust all conclusions based on model predictions. In the context of moment arms, muscle-DOFs that include reasonable measured moment arm data (with or without merging datasets), can lead to false conclusions about errors in torque production. Associated errors might prompt modification to musculotendon forcegeneration properties to compensate for these errors when small adjustments in the muscle path geometry (and resulting moment arm) could correct them. In other words, without properly identifying or considering all the factors affecting the desired output(s), alterations to other parameters may be unknowingly moved into unphysiological values due to misclassifying or addressing the true underlying error(s). That said, the limitation of small disparate subsets of measured data reiterates the need for expanding limited and unstandardized musculoskeletal validation procedures and tools.

\subsection{Future Work}

Future development for the work discussed in this thesis would include improving the developed software tools for moment arm digitization and storage and implementing appropriate changes to the identified errors in the evaluated musculoskeletal model.

The classes used to extract all the WebPlotDigitizer (Rohatgi, 2019) datasets containing the published moment arm data could be less coupled (i.e., dependent) on one another while maintaining cohesiveness. This would allow users to utilize individual classes and features 
independently within or outside the presented workflow. Furthermore, improvements to the computational speed and size requirements of the base classes would greatly reduce the amount of RAM need to manipulate and access larger datasets. Specifically, this would provide more flexibility in how physical data is stored locally or remotely and decrease hardware performance requirements needed to use the software (i.e., could be used on a personal laptop). The development of user-interfaces would drastically improve the end-user experience, allowing them to visually interact with different objects and datasets within a table or database. This would particularly benefit less experienced users. This would also enhance productivity by decreasing the number of interactions needed to perform a specific task and more visuals to better troubleshoot errors. Another major improvement would be developing additional features - this would aim to expand the toolbox capabilities by including additional packages or extensions similar to other musculoskeletal software packages (Damsgaard et al., 2006; Scott L. Delp et al., 2007; Pizzolato et al., 2015; Seth et al., 2018; Valente et al., 2017). In regards to the content of this thesis, additional tools could include moment arm validation frameworks (Boots et al., 2020), curve-fitting for high-dimensional data (Sartori et al., 2012; Sobinov et al., 2019), and optimizers for muscle-path adjustments (Carbone et al., 2012; Pellikaan et al., 2014). With these discussed improvements, the potential for more high-quality musculoskeletal data can be stored and created for model validation.

Future work with the created muscle-DOF relationships from measured datasets would be used to fully validate the evaluated model in this study. By initially evaluating the (Lai et al., 2017) model, we would implement the necessary changes to problematic muscle path geometry and correct poor moment arm profiles. This would address the first 2 steps described in (Boots et al., 2020). The third step would require obtaining maximum isometric torque measurements for the hip, knee, and ankle joints so that we could properly scale the maximum force-generation capacity of the model to physiologically valid torque values. Because the torque scaling step uses scalar 
values, future works would need to determine the most appropriate posture and torque value used to scale between the measured and simulated torques. This would prove to be a crucial step due to the postural dependence of moment arms and subsequent muscle torques (Gonzalez et al., 1997). Correspondingly, the resulting model would need to undergo several indirect validation comparisons to ensure that the model's overall function is valid and whether it represents a generic individual. The additional tools proposed for future work in the moment arm toolbox above would greatly improve the validation process by further automating tedious and time-consuming components of the musculoskeletal development process.

\section{Chapter 5 Conclusion}

In my thesis, I developed a computational workflow for digitizing posture-dependent moment arm datasets. I also detail the methods and tools used for creating and managing a custom relational database for the digitized datasets to create moment arm profiles that captured the accuracy and viability of multiple datasets to validate a human lower limb musculoskeletal model. I obtained a previously published human lower limb musculoskeletal model to evaluate its muscle-DOF relationships using a validation framework for musculoskeletal models. I used the described validation process for a generic lower limb musculoskeletal model and used the created moment arm profiles as the gold standard for comparison to assess structural and functional errors in muscle path geometry and moment arm relationships within the model. Here, I identify errors in developing and validating these models and demonstrate a new approach for creating more reliable and generic musculoskeletal models. Overall, my work addresses and solves the need for standardized tools for managing morphological datasets for comparison and analysis, which can be used to improve development and validation efforts in musculoskeletal modeling. 


\section{References}

An, K. N., Takahashi, K., Harrigan, T. P., \& Chao, E. Y. (1984). Determination of Muscle Orientations and Moment Arms. Journal of Biomechanical Engineering, 106(3), 280-282. https://doi.org/10.1115/1.3138494

An, K. N., Ueba, Y., Chao, E. Y., Cooney, W. P., \& Linscheid, R. L. (1983). Tendon excursion and moment arm of index finger muscles. Journal of Biomechanics, 16(6), 419-425. https://doi.org/10.1016/0021-9290(83)90074-X

Arnold, A. S., \& Delp, S. L. (2001). Rotational moment arms of the medial hamstrings and adductors vary with femoral geometry and limb position: Implications for the treatment of internally rotated gait. Journal of Biomechanics, 34(4), 437-447. https://doi.org/10.1016/S0021-9290(00)00232-3

Arnold, A. S., Salinas, S., Hakawa, D. J., \& Delp, S. L. (2000). Accuracy of Muscle Moment Arms Estimated from MRI-Based Musculoskeletal Models of the Lower Extremity. Computer Aided Surgery, 5(2), 108-119. https://doi.org/10.3109/10929080009148877

Arnold, A. S., Thelen, D. G., Schwartz, M. H., Anderson, F. C., \& Delp, S. L. (2007). Muscular coordination of knee motion during the terminal-swing phase of normal gait. Journal of Biomechanics, 40(15), 3314-3324. https://doi.org/10.1016/j.jbiomech.2007.05.006

Arnold, E. M., Ward, S. R., Lieber, R. L., \& Delp, S. L. (2010). A Model of the Lower Limb for Analysis of Human Movement. Annals of Biomedical Engineering, 38(2), 269-279. https://doi.org/10.1007/s10439-009-9852-5

Bernstein, N. A. (1967). The co-ordination and regulation of movements. Oxford: Pergamon Press. 
Blemker, S. S., \& Delp, S. L. (2005). Three-Dimensional Representation of Complex Muscle Architectures and Geometries. Annals of Biomedical Engineering, 33(5), 661-673. https://doi.org/10.1007/s10439-005-1433-7

Boots, M. T., Hardesty, R., Sobinov, A., Gritsenko, V., Collinger, J. L., Fisher, L. E., Gaunt, R., \& Yakovenko, S. (2020). Functional and Structural Moment Arm Validation for Musculoskeletal Models: A Study of the Human Forearm and Hand. BioRxiv, 2020.05.29.124644. https://doi.org/10.1101/2020.05.29.124644

Buford, William L, \& Andersen, C. R. (2006). Predicting Moment Arms in Diarthroidal Joints - 3D Computer Simulation Capability and Muscle-Tendon Model Validation. 4.

Buford, William L, Ivey, F. M., Nakamura, T., \& Nguyen, D. K. (2001). Internal/external rotation moment arms of muscles at the knee: Moment arms for the normal knee and the ACLdeficient knee. 11.

Buford, W.L., Ivey, F. M., Malone, J. D., Patterson, R. M., Pearce, G. L., Nguyen, D. K., \& Stewart, A. A. (1997). Muscle balance at the knee-moment arms for the normal knee and the ACLminus knee. IEEE Transactions on Rehabilitation Engineering, 5(4), 367-379. https://doi.org/10.1109/86.650292

Bujalski, P., Martins, J., \& Stirling, L. (2018). A Monte Carlo analysis of muscle force estimation sensitivity to muscle-tendon properties using a Hill-based muscle model. Journal of Biomechanics, 79, 67-77. https://doi.org/10.1016/j.jbiomech.2018.07.045

Burger, B., \& Toiviainen, P. (2013). MoCap Toolbox-A MATLAB Toolbox for Computational Analysis of Movement. 8.

Carbone, V., Fluit, R., Pellikaan, P., van der Krogt, M. M., Janssen, D., Damsgaard, M., Vigneron, L., Feilkas, T., Koopman, H. F. J. M., \& Verdonschot, N. (2015). TLEM 2.0 - A comprehensive musculoskeletal geometry dataset for subject-specific modeling of lower 
extremity. Journal of Biomechanics, 48(5), 734-741. https://doi.org/10.1016/j.jbiomech.2014.12.034

Carbone, V., van der Krogt, M. M., Koopman, H. F. J. M., \& Verdonschot, N. (2012). Sensitivity of subject-specific models to errors in musculo-skeletal geometry. Journal of Biomechanics, 45(14), 2476-2480. https://doi.org/10.1016/j.jbiomech.2012.06.026

Chvatal, S. A., \& Ting, L. H. (2013). Common muscle synergies for balance and walking. Frontiers in Computational Neuroscience, 7. https://doi.org/10.3389/fncom.2013.00048

Cook, D., Julias, M., \& Nauman, E. (2014). Biological variability in biomechanical engineering research: Significance and meta-analysis of current modeling practices. Journal of Biomechanics, 47(6), 1241-1250. https://doi.org/10.1016/j.jbiomech.2014.01.040

Crouch, D. L., \& Huang, H. (2016). Lumped-parameter electromyogram-driven musculoskeletal hand model: A potential platform for real-time prosthesis control. Journal of Biomechanics, 49(16), 3901-3907. https://doi.org/10.1016/j.jbiomech.2016.10.035

Crowninshield, R. D. (1981). A physiologically based criterion of muscle force prediction in locomotion. Journal of Biomechanics, 14(11), 793-801.

Damsgaard, M., Rasmussen, J., Christensen, S. T., Surma, E., \& de Zee, M. (2006). Analysis of musculoskeletal systems in the AnyBody Modeling System. Simulation Modelling Practice and Theory, 14(8), 1100-1111. https://doi.org/10.1016/j.simpat.2006.09.001

Delp, Scott L., Anderson, F. C., Arnold, A. S., Loan, P., Habib, A., John, C. T., Guendelman, E., \& Thelen, D. G. (2007). OpenSim: Open-Source Software to Create and Analyze Dynamic Simulations of Movement. IEEE Transactions on Biomedical Engineering, 54(11), 19401950. https://doi.org/10.1109/TBME.2007.901024 
Delp, Scott L, Hess, W. E., Hungerford, D. S., \& Jones, L. C. (1999). Variation of rotation moment arms with hip flexion. Journal of Biomechanics, 32(5), 493-501. https://doi.org/10.1016/S0021-9290(99)00032-9

Delp, Scott L., \& Loan, J. P. (1995). A graphics-based software system to develop and analyze models of musculoskeletal structures. Computers in Biology and Medicine, 25(1), 21-34. https://doi.org/10.1016/0010-4825(95)98882-E

Delp, Scott L., Ringwelski, D. A., \& Carroll, N. C. (1994). Transfer of the rectus femoris: Effects of transfer site on moment arms about the knee and hip. Journal of Biomechanics, 27(10), 1201-1211. https://doi.org/10.1016/0021-9290(94)90274-7

Delp, S.L., Loan, J. P., Hoy, M. G., Zajac, F. E., Topp, E. L., \& Rosen, J. M. (1990). An interactive graphics-based model of the lower extremity to study orthopaedic surgical procedures. IEEE Transactions on Biomedical Engineering, 37(8), 757-767. https://doi.org/10.1109/10.102791

DeMers, M. S., Pal, S., \& Delp, S. L. (2014). Changes in tibiofemoral forces due to variations in muscle activity during walking: TIBIOFEMORAL FORCES AND MUSCLE ACTIVITY. Journal of Orthopaedic Research, 32(6), 769-776. https://doi.org/10.1002/jor.22601

Dixon, P. C., Loh, J. J., Michaud-Paquette, Y., \& Pearsall, D. J. (2017). biomechZoo: An opensource toolbox for the processing, analysis, and visualization of biomechanical movement data. Computer Methods and Programs in Biomedicine, 140, 1-10. https://doi.org/10.1016/j.cmpb.2016.11.007

Dostal, W. F., Soderberg, G. L., \& Andrews, J. G. (1986). Actions of hip muscles. Physical Therapy, 66(3), 351-361. 
Draganich, L. F., Andriacchi, T. P., \& Andersson, G. B. J. (1987). Interaction between intrinsic knee mechanics and the knee extensor mechanism. Journal of Orthopaedic Research, 5(4), 539-547. https://doi.org/10.1002/jor.1100050409

Duda, G. N., Brand, D., Freitag, S., Lierse, W., \& Schneider, E. (1996). Variability of femoral muscle attachments. Journal of Biomechanics, 29(9), 1185-1190. https://doi.org/10.1016/0021-9290(96)00025-5

Durandau, G., Farina, D., \& Sartori, M. (2018). Robust Real-Time Musculoskeletal Modeling Driven by Electromyograms. IEEE Transactions on Biomedical Engineering, 65(3), 556564. https://doi.org/10.1109/TBME.2017.2704085

Eng, C. M., Arnold, A. S., Lieberman, D. E., \& Biewener, A. A. (2015). The capacity of the human iliotibial band to store elastic energy during running. Journal of Biomechanics, 48(12), 3341-3348. https://doi.org/10.1016/j.jbiomech.2015.06.017

Erdemir, A., McLean, S., Herzog, W., \& van den Bogert, A. J. (2007). Model-based estimation of muscle forces exerted during movements. Clinical Biomechanics, 22(2), 131-154. https://doi.org/10.1016/j.clinbiomech.2006.09.005

Fath, F., Blazevich, A. J., Waugh, C. M., Miller, S. C., \& Korff, T. (2010). Direct comparison of in vivo Achilles tendon moment arms obtained from ultrasound and MR scans. Journal of Applied Physiology, 109(6), 1644-1652. https://doi.org/10.1152/japplphysiol.00656.2010

Goislard De Monsabert, B., Edwards, D., Shah, D., \& Kedgley, A. (2018). Importance of Consistent Datasets in Musculoskeletal Modelling: A Study of the Hand and Wrist. Annals of Biomedical Engineering, 46(1), 71-85. https://doi.org/10.1007/s10439-017-1936-z

Gonzalez, R. V., Buchanan, T. S., \& Delp, S. L. (1997). How muscle architecture and moment arms affect wrist flexion-extension moments. Journal of Biomechanics, 30(7), 705-712. https://doi.org/10.1016/S0021-9290(97)00015-8 
Graczyk, E. L., Resnik, L., Schiefer, M. A., Schmitt, M. S., \& Tyler, D. J. (2018). Home Use of a Neural-connected Sensory Prosthesis Provides the Functional and Psychosocial Experience of Having a Hand Again. Scientific Reports, 8(1), 9866. https://doi.org/10.1038/s41598-018-26952-x

Grood, E. S., Suntay, W. J., Noyes, F. R., \& Butler, D. L. (1984). Biomechanics of the KneeExtension Exercise. The Journal of Bone and Joint Surgery, 66(5).

Hamner, S. R., Seth, A., \& Delp, S. L. (2010). Muscle contributions to propulsion and support during running. Journal of Biomechanics, 43(14), 2709-2716. https://doi.org/10.1016/j.jbiomech.2010.06.025

Handsfield, G. G., Meyer, C. H., Hart, J. M., Abel, M. F., \& Blemker, S. S. (2014). Relationships of 35 lower limb muscles to height and body mass quantified using MRI. Journal of Biomechanics, 47(3), 631-638. https://doi.org/10.1016/j.jbiomech.2013.12.002

Haugstvedt, J. R., Berger, R. A., \& Berglund, L. J. (2001). A mechanical study of the momentforces of the supinators and pronators of the forearm. Acta Orthopaedica Scandinavica. https://doi.org/10.1080/000164701317269076

Hawkins, D. (1992). Software for determining lower extremity muscle-tendon kinematics and moment arm lengths during flexion/extension movements. Computers in Biology and Medicine, 22(1), 59-71. https://doi.org/10.1016/0010-4825(92)90052-O

He Huang, Ping Zhou, Guanglin Li, \& Kuiken, T. A. (2008). An Analysis of EMG Electrode Configuration for Targeted Muscle Reinnervation Based Neural Machine Interface. IEEE Transactions on Neural Systems and Rehabilitation Engineering, 16(1), 37-45. https://doi.org/10.1109/TNSRE.2007.910282

Henneman, E. (1957). Relation between size of neurons and their susceptibility to discharge. Science, 126(3287), 1345-1347. https://doi.org/10.1126/science.126.3287.1345 
Henninger, H. B., Reese, S. P., Anderson, A. E., \& Weiss, J. A. (2010). Validation of computational models in biomechanics. Proceedings of the Institution of Mechanical Engineers, Part H: Journal of Engineering in Medicine, 224(7), 801-812. https://doi.org/10.1243/09544119JEIM649

Herzog, W, \& Read, L. J. (1993). Lines of action and moment arms of the major force-carrying structures crossing the human knee joint. Journal of Anatomy, 182(Pt 2), 213-230.

Herzog, Walter. (1992). Sensitivity to Muscle Force Estimations to Changes in Muscle Input Parameters Using Nonlinear Optimization Approaches. Journal of Biomechanical Engineering, 114(2), 267-268.

Hicks, J. L., Uchida, T. K., Seth, A., Rajagopal, A., \& Delp, S. L. (2015). Is My Model Good Enough? Best Practices for Verification and Validation of Musculoskeletal Models and Simulations of Movement. Journal of Biomechanical Engineering, 137(2), 020905. https://doi.org/10.1115/1.4029304

Hill, A. V. (1938). The heat of shortening and the dynamic constants of muscle. Proceedings of the Royal Society of London. Series B: Biological Sciences, 126, 60. https://doi.org/10.1098/rspb.1938.0050

Horsman, Klein, M. D., Koopman, H. F. J. M., van der Helm, F. C. T., Prosé, L. P., \& Veeger, H. E. J. (2007). Morphological muscle and joint parameters for musculoskeletal modelling of the lower extremity. Clinical Biomechanics, 22(2), 239-247. https://doi.org/10.1016/j.clinbiomech.2006.10.003

Hunter, S. K., Pereira, H. M., \& Keenan, K. G. (2016). The aging neuromuscular system and motor performance. Journal of Applied Physiology, 121(4), 982-995. https://doi.org/10.1152/japplphysiol.00475.2016

Huwaldt, J. (2018). Plot Digitizer. http://plotdigitizer.sourceforge.net/ 
Huxley, A. F. (1957). Muscle structure and theories of contraction. Progress in Biophysics and Biophysical Chemistry, 7, 255-318.

Ito, M., Akima, H., \& Fukunaga, T. (2000). In vivo moment arm determination using B-mode ultrasonography. Journal of Biomechanics, 33(2), 215-218. https://doi.org/10.1016/S0021-9290(99)00154-2

jiro. (2020). GRABIT (1.0.0.1) [Computer software]. jiro (2020). GRABIT (https://www.mathworks.com/matlabcentral/fileexchange/7173-grabit), MATLAB Central File Exchange. Retrieved June 17, 2020.

Klein, P., Mattys, S., \& Rooze, M. (1996). Moment arm length variations of selected muscles acting on talocrural and subtalar joints during movement: An In vitro study. Journal of Biomechanics, 29(1), 21-30. https://doi.org/10.1016/0021-9290(95)00025-9

Kuiken, T. A. (2009). Targeted Muscle Reinnervation for Real-time Myoelectric Control of Multifunction Artificial Arms. JAMA, 301(6), 619. https://doi.org/10.1001/jama.2009.116

Lai, A. K. M., Arnold, A. S., \& Wakeling, J. M. (2017). Why are Antagonist Muscles Co-activated in My Simulation? A Musculoskeletal Model for Analysing Human Locomotor Tasks. Annals of Biomedical Engineering, 45(12), 2762-2774. https://doi.org/10.1007/s10439017-1920-7

Landsmeer, J. M. (1961). Studies in the anatomy of articulation. I. The equilibrium of the “intercalated” bone. Acta Morphologica Neerlando-Scandinavica, 3, 287-303.

Laurent, K., De Sèze, M.-P., Delleci, C., Koleck, M., Dehail, P., Orgogozo, J.-M., \& Mazaux, J.M. (2011). Assessment of quality of life in stroke patients with hemiplegia. Annals of Physical and Rehabilitation Medicine, 54(6), 376-390. https://doi.org/10.1016/j.rehab.2011.06.002 
Leardini, A., \& O'Connor, J. J. (2002). A model for lever-arm length calculation of the flexor and extensor muscles at the ankle. Gait \& Posture, 15(3), 220-229. https://doi.org/10.1016/S0966-6362(01)00153-9

Lee, S. S. M., \& Piazza, S. J. (2008). Inversion-eversion moment arms of gastrocnemius and tibialis anterior measured in vivo. Journal of Biomechanics, 41(16), 3366-3370. https://doi.org/10.1016/j.jbiomech.2008.09.029

Lengsfeld, M., Pressel, T., \& Stammberger, U. (1997). Lengths and lever arms of hip joint muscles: Geometrical analyses using a human multibody model. Gait \& Posture, 6(1), 1826. https://doi.org/10.1016/S0966-6362(96)01100-9

Lieber, R. L. (2000). Functional and clinical significance of skeletal muscle architecture. 20.

Loren, G. J., Shoemaker, S. D., Burkholder, T. J., Jacobson, M. D., Fridén, J., \& Lieber, R. L. (1996). Human wrist motors: Biomechanical design and application to tendon transfers. Journal of Biomechanics, 29(3), 331-342. https://doi.org/10.1016/0021-9290(95)00055-0

Lund, M. E., de Zee, M., Andersen, M. S., \& Rasmussen, J. (2012). On validation of multibody musculoskeletal models. Proceedings of the Institution of Mechanical Engineers, Part H: Journal of Engineering in Medicine, 226(2), 82-94. https://doi.org/10.1177/0954411911431516

Maas, S. A., Ellis, B. J., Ateshian, G. A., \& Weiss, J. A. (2012). FEBio: Finite Elements for Biomechanics. Journal of Biomechanical Engineering, 134(1), 011005. https://doi.org/10.1115/1.4005694

Maganaris, C. N. (2001). Force-length characteristics of in vivo human skeletal muscle. Acta Physiologica Scandinavica, 172(4), 279-285. https://doi.org/10.1046/j.1365201x.2001.00799.x 
Maganaris, Constantinos N. (2000). In vivo measurement-based estimations of the moment arm in the human tibialis anterior muscle-tendon unit. Journal of Biomechanics, 33(3), 375379. https://doi.org/10.1016/S0021-9290(99)00188-8

Maganaris, Constantinos N. (2004). Imaging-based estimates of moment arm length in intact human muscle-tendons. European Journal of Applied Physiology, 91(2-3), 130-139. https://doi.org/10.1007/s00421-003-1033-x

Maganaris, Constantinos N., Baltzopoulos, V., \& Sargeant, A. J. (1998). Changes in Achilles tendon moment arm from rest to maximum isometric plantarflexion: In vivo observations in man. The Journal of Physiology, 510(3), 977-985. https://doi.org/10.1111/j.14697793.1998.977bj.x

Maganaris, Constantinos N, Baltzopoulos, V., \& Sargeant, A. J. (1999). Changes in the tibialis anterior tendon moment arm from rest to maximum isometric dorsi-exion: In vivo observations in man. Clinical Biomechanics, 6.

Maganaris, Constantinos N., Baltzopoulos, V., \& Sargeant, A. J. (2000). In vivo measurementbased estimations of the human Achilles tendon moment arm. European Journal of Applied Physiology, 83(4-5), 363-369. https://doi.org/10.1007/s004210000247

Magermans, D. J., Chadwick, E. K. J., Veeger, H. E. J., Rozing, P. M., \& van der Helm, F. C. T. (2004). Effectiveness of tendon transfers for massive rotator cuff tears: A simulation study. Clinical Biomechanics, 19(2), 116-122. https://doi.org/10.1016/j.clinbiomech.2003.09.008

Mantoan, A., Pizzolato, C., Sartori, M., Sawacha, Z., Cobelli, C., \& Reggiani, M. (2015). MOtoNMS: A MATLAB toolbox to process motion data for neuromusculoskeletal modeling and simulation. Source Code for Biology and Medicine, 10(1), 12. https://doi.org/10.1186/s13029-015-0044-4 
McCullough, M. B. A., Ringleb, S. I., Arai, K., Kitaoka, H. B., \& Kaufman, K. R. (2011). Moment Arms of the Ankle Throughout the Range of Motion in Three Planes. Foot \& Ankle International, 32(3), 300-306. https://doi.org/10.3113/FAI.2011.0300

McKay, J. L., \& Ting, L. H. (2012). Optimization of Muscle Activity for Task-Level Goals Predicts Complex Changes in Limb Forces across Biomechanical Contexts. PLoS Computational Biology, 8(4), e1002465. https://doi.org/10.1371/journal.pcbi.1002465

Millard, M., Uchida, T., Seth, A., \& Delp, S. L. (2013). Flexing Computational Muscle: Modeling and Simulation of Musculotendon Dynamics. Journal of Biomechanical Engineering, 135(2), 021005. https://doi.org/10.1115/1.4023390

Modenese, L., Montefiori, E., Wang, A., Wesarg, S., Viceconti, M., \& Mazzà, C. (2018). Investigation of the dependence of joint contact forces on musculotendon parameters using a codified workflow for image-based modelling. Journal of Biomechanics, 73, 108118. https://doi.org/10.1016/j.jbiomech.2018.03.039

Nemeth, G., \& Ohlsen, H. (1985). In vivo moment arm lengths for hip extensor muscles at different angles of hip flexion. Journal of Biomechanics, 18(2), 129-140. https://doi.org/10.1016/0021-9290(85)90005-3

Nemeth, G., \& Ohlsen, H. (1989). Moment arms of hip abductor and adductor muscles measured in vivo by computed tomography. Clinical Biomechanics, 4(3), 133-136. https://doi.org/10.1016/0268-0033(89)90015-6

Neptune, R. R., Kautz, S. A., \& Zajac, F. E. (2001). Contributions of the individual ankle plantar flexors to support, forward progression and swing initiation during walking. Journal of Biomechanics, 34(11), 1387-1398. https://doi.org/10.1016/S0021-9290(01)00105-1

Nolte, D., Tsang, C. K., Zhang, K. Y., Ding, Z., Kedgley, A. E., \& Bull, A. M. J. (2016). Non-linear scaling of a musculoskeletal model of the lower limb using statistical shape models. 
Journal of Biomechanics, $\quad$ 49(14), 3576-3581. https://doi.org/10.1016/j.jbiomech.2016.09.005

Nussbaum, M., Chaffin, D., \& Rechtien, C. (1995). Muscle lines-of-action affect predicted forces in optimization-based spine muscle modeling. Journal of Biomechanics, 28(4), 9.

Pellikaan, P., van der Krogt, M. M., Carbone, V., Fluit, R., Vigneron, L. M., Van Deun, J., Verdonschot, N., \& Koopman, H. F. J. M. (2014). Evaluation of a morphing based method to estimate muscle attachment sites of the lower extremity. Journal of Biomechanics, 47(5), 1144-1150. https://doi.org/10.1016/j.jbiomech.2013.12.010

Piazza, S. J., Adamson, R. L., Sanders, J. O., \& Sharkey, N. A. (2001). Changes in muscle moment arms following split tendon transfer of tibialis anterior and tibialis posterior. Gait \& Posture, 14(3), 271-278. https://doi.org/10.1016/S0966-6362(01)00143-6

Pizzolato, C., Lloyd, D. G., Sartori, M., Ceseracciu, E., Besier, T. F., Fregly, B. J., \& Reggiani, M. (2015). CEINMS: A toolbox to investigate the influence of different neural control solutions on the prediction of muscle excitation and joint moments during dynamic motor tasks. Journal of Biomechanics, 48(14), 3929-3936. https://doi.org/10.1016/j.jbiomech.2015.09.021

Rajagopal, A., Dembia, C. L., DeMers, M. S., Delp, D. D., Hicks, J. L., \& Delp, S. L. (2016). FullBody Musculoskeletal Model for Muscle-Driven Simulation of Human Gait. IEEE Transactions on Biomedical Engineering, 63(10), 2068-2079. https://doi.org/10.1109/TBME.2016.2586891

Reuleaux, F. (1876). The Kinematics of Machinery: Outlines of a Theory of Machines. London, Macmillan.

Richards, R. E., Andersen, M. S., Harlaar, J., \& van den Noort, J. C. (2018). Relationship between knee joint contact forces and external knee joint moments in patients with medial knee 
osteoarthritis: Effects of gait modifications. Osteoarthritis and Cartilage, 26(9), 12031214. https://doi.org/10.1016/j.joca.2018.04.011

Rohatgi, A. (2019). WebPlotDigitizer (4.2) [Computer software]. https://automeris.io/WebPlotDigitizer

Romero, F., \& Alonso, F. J. (2016). A comparison among different Hill-type contraction dynamics formulations for muscle force estimation. Mechanical Sciences, 7(1), 19-29. https://doi.org/10.5194/ms-7-19-2016

Roser, M., Ortiz-Ospina, E., \& Ritchie, H. (2020). Life Expectancy. Our World in Data. https://ourworldindata.org/life-expectancy

Rugg, S. G., Gregor, R. J., Mandelbaum, B. R., \& Chiu, L. (1990). In vivo moment arm calculations at the ankle using magnetic resonance imaging (MRI). Journal of Biomechanics, 23(5), 495-501.

Sartori, M., Durandau, G., Došen, S., \& Farina, D. (2018). Robust simultaneous myoelectric control of multiple degrees of freedom in wrist-hand prostheses by real-time neuromusculoskeletal modeling. Journal of Neural Engineering, 15(6), 066026. https://doi.org/10.1088/1741-2552/aae26b

Sartori, M., Reggiani, M., Farina, D., \& Lloyd, D. G. (2012). EMG-Driven Forward-Dynamic Estimation of Muscle Force and Joint Moment about Multiple Degrees of Freedom in the Human Lower Extremity. PLoS ONE, 7(12), e52618. https://doi.org/10.1371/journal.pone.0052618

Scheys, L., Desloovere, K., Suetens, P., \& Jonkers, I. (2011). Level of subject-specific detail in musculoskeletal models affects hip moment arm length calculation during gait in pediatric subjects with increased femoral anteversion. Journal of Biomechanics, 44(7), 1346-1353. https://doi.org/10.1016/j.jbiomech.2011.01.001 
Scovil, C. Y., \& Ronsky, J. L. (2006). Sensitivity of a Hill-based muscle model to perturbations in model parameters. Journal of Biomechanics, 39(11), 2055-2063. https://doi.org/10.1016/j.jbiomech.2005.06.005

Seth, A., Hicks, J. L., Uchida, T. K., Dembia, C. L., Rajagopal, A., Habib, A., Dunne, J. J., Ong, C. F., DeMers, M. S., Millard, M., Hamner, S. R., Arnold, E. M., Yong, J. R., Lakshmikanth, S. K., Sherman, M. A., Ku, J. P., \& Delp, S. L. (2018). OpenSim: Simulating musculoskeletal dynamics and neuromuscular control to study human and animal

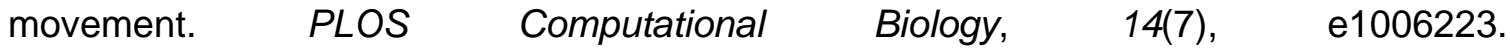
https://doi.org/10.1371/journal.pcbi.1006223

Sobinov, A. (2019). Description of motor control using inverse models [PhD, West Virginia University Libraries]. https://doi.org/10.33915/etd.4073

Sobinov, A., Boots, M. T., Gritsenko, V., Fisher, L. E., Gaunt, R. A., \& Yakovenko, S. (2019). Approximating complex musculoskeletal biomechanics using multidimensional autogenerating polynomials. BioRxiv. https://doi.org/10.1101/759878

Spoor, C. W., \& van Leeuwen, J. L. (1992). Knee muscle moment arms from MRI and from tendon travel. Journal of Biomechanics, 25(2), 201-206.

Spoor, C. W., van Leeuwen, J. L., Meskers, C. G. M., Titulaer, A. F., \& Huson, A. (1990). Estimation of instantaneous moment arms of lower-leg muscles. Journal of Biomechanics, 23(12), 1247-1259.

Steele, K. M., Jackson, R. W., Shuman, B. R., \& Collins, S. H. (2017). Muscle recruitment and coordination with an ankle exoskeleton. Journal of Biomechanics, 59, 50-58. https://doi.org/10.1016/j.jbiomech.2017.05.010 
Thelen, D. G. (2003). Adjustment of Muscle Mechanics Model Parameters to Simulate Dynamic Contractions in Older Adults. Journal of Biomechanical Engineering, 125(1), 70-77. https://doi.org/10.1115/1.1531112

Thelen, D. G., Chumanov, E. S., Hoerth, D. M., Best, T. M., Swanson, S. C., Li, L., Young, M., \& Heiderscheit, B. C. (2005). Hamstring Muscle Kinematics during Treadmill Sprinting: Medicine \& Science in Sports \& Exercise, 37(1), 108-114. https://doi.org/10.1249/01.MSS.0000150078.79120.C8

Todorov, E., Erez, T., \& Tassa, Y. (2012). MuJoCo: A physics engine for model-based control. 2012 IEEE/RSJ International Conference on Intelligent Robots and Systems, 5026-5033. https://doi.org/10.1109/IROS.2012.6386109

Vaarbakken, K., Steen, H., Samuelsen, G., Dahl, H. A., Leergaard, T. B., \& Stuge, B. (2015). Primary functions of the quadratus femoris and obturator externus muscles indicated from lengths and moment arms measured in mobilized cadavers. Clinical Biomechanics, 30(3), 231-237. https://doi.org/10.1016/j.clinbiomech.2015.02.004

Valente, G., Crimi, G., Vanella, N., Schileo, E., \& Taddei, F. (2017). nmsBuilder: Freeware to create subject-specific musculoskeletal models for OpenSim. Computer Methods and Programs in Biomedicine, 152, 85-92. https://doi.org/10.1016/j.cmpb.2017.09.012

van der Helm, F. C. T., \& Veenbaas, R. (1991). Modelling the mechanical effect of muscles with large attachment sites: Application to the shoulder mechanism. Journal of Biomechanics, 24(12), 1151-1163.

van der Krogt, M. M., Delp, S. L., \& Schwartz, M. H. (2012). How robust is human gait to muscle weakness? Gait \& Posture, 36(1), 113-119. https://doi.org/10.1016/j.gaitpost.2012.01.017 
van Soest, A. J., \& Bobbert, M. F. (1993). The contribution of muscle properties in the control of explosive movements. Biological Cybernetics, 69(3), 195-204. https://doi.org/10.1007/BF00198959

Visser, J. J., Hoogkamer, J. E., Bobbert, M. F., \& Huijing, P. A. (1990). Length and moment arm of human leg muscles as a function of knee and hip-joint angles. European Journal of Applied Physiology and Occupational Physiology, 61(5-6), 453-460. https://doi.org/10.1007/BF00236067

Ward, S. R., Eng, C. M., Smallwood, L. H., \& Lieber, R. L. (2009). Are Current Measurements of Lower Extremity Muscle Architecture Accurate? Clinical Orthopaedics and Related Research, 467(4), 1074-1082. https://doi.org/10.1007/s11999-008-0594-8

Winby, C. R., Lloyd, D. G., \& Kirk, T. B. (2008). Evaluation of different analytical methods for subject-specific scaling of musculotendon parameters. Journal of Biomechanics, 41(8), 1682-1688. https://doi.org/10.1016/j.jbiomech.2008.03.008

Wretenberg, P., Nemeth, G., Lamontagne, M., \& Lundin, B. (1996). Passive knee muscle moment arms measured in vivo with MRI. Clinical Biomechanics, 11(8), 439-446. https://doi.org/10.1016/S0268-0033(96)00030-7

Yamaguchi, G. T. (2001). Dynamic Modeling of Musculoskeletal Motion. Springer. https://doi.org/10.1007\%2F978-0-387-28750-8

Yamaguchi, G. T., \& Zajac, F. E. (1989). A planar model of the knee joint to characterize the knee extensor mechanism. Journal of Biomechanics, 22(1), 1-10. https://doi.org/10.1016/00219290(89)90179-6

Zajac, F.E. (1989). Muscle and tendon: Properties, models, scaling, and application to biomechanics and motor control. Critical Reviews in Biomedical Engineering, 17(4), 359411. 
Zajac, Felix E. (1993). Muscle coordination of movement: A perspective. Journal of Biomechanics, 26, 109-124. https://doi.org/10.1016/0021-9290(93)90083-Q 